\title{
Introduction to Periodic Homogenization
}

\author{
François ALOUGES*
}

CMAP, Ecole polytechnique, CNRS, Universite Paris-Saclay, 91128, Palaiseau, France

These notes contain an introduction of the theory of multiscale analysis and periodic homogenization of PDEs. Basic tools, such as multiscale expansion, 2-scale convergence, and Gamma-convergence are introduced and carefully explained. Application to diffusion equation or porous media are also considered, as well as numerical methods. Exercises are also provided to help the reader to become familiar with the theory.

KEYWORDS: Homogenization, multiscale expansion, 2-scale convergence, porous media, Gammaconvergence

\section{Contents}

1 Formal Asymptotic Homogenization 149

1.1 Introduction . . . . . . . . . . . . . . . . . . . . . . . . . . . . 149

1.2 The classical setting ... . . . . . . . . . . . . . . . . . . . 149

1.3 Multiscale expansion ... . . . . . . . . . . . . . . . . . 150

1.4 The cell and the homogenized problems . . . . . . . . . . . . . . . . . . . . . . . . . . . . . . . . .

1.5 Exercises . . . . . . . . . . . . . . . . . . . . 153

2 Two-scale Convergence $\quad 154$

2.1 Introduction . . . . . . . . . . . . . . . . . . . . . . . . 154

2.2 Two-scale convergence . . . . . . . . . . . . . . . . . . . . 154

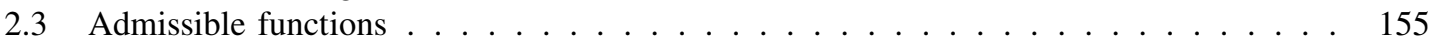

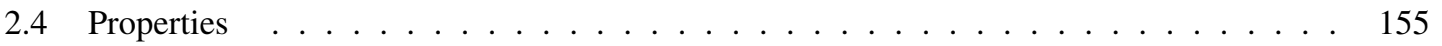

2.5 Exercises . . . . . . . . . . . . . . . . . . . . . . . . 157

3 Application to Linear Elliptic Equations $\quad 158$

3.1 Homogenization of 2 nd order elliptic problems . . . . . . . . . . . . . . . . . 158

3.2 Existence and uniqueness . . . . . . . . . . . . . . . . . . . . 159

3.3 The cell and the homogenized problems . . . . . . . . . . . . . . . . 160

3.4 Exercises ............................ 161

4 Convergence of the Energy $\quad 161$

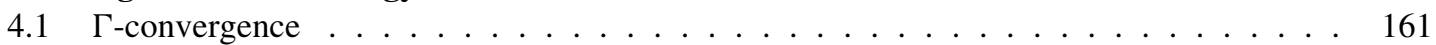

4.2 Application to homogenization . . . . . . . . . . . . . . . . . 163

4.3 Exercises . . . . . . . . . . . . . . . . . . . . 165

5 Perforated Domains - Porous Media 166

5.1 Setting of the problem ....................... 166

5.2 Homogenization . . . . . . . . . . . . . . . . . . . . . . 167

5.3 Convergence Theorem . . . . . . . . . . . . . . . . . . . . . 168

5.4 The cell and the homogenized problem . . . . . . . . . . . . . . . 171

5.5 Exercises . . . . . . . . . . . . . . . . . . . . . 172

6 Numerical Methods in Homogenization $\quad 172$

6.1 Classical error estimates . . . . . . . . . . . . . . . . . . . . . . 172

6.2 Application to homogenization problems . . . . . . . . . . . . . . . . 176

6.3 The multiscale finite element method (MFEM) . . . . . . . . . . . . . . . . 182

6.4 Conclusion . . . . . . . . . . . . . . . . . . . . 186 


\section{Foreword}

These notes contain the material that was used for a one week minicourse given by the author during the summer of 2015 in Tohoku University, in Sendai. The author would like to thank very much the organizers of the mini-course, and especially Reika Fukuizumi, Jun Masamune and Shigeru Sakaguchi for their very kind hospitality and the opportunity they gave him to come to Japan.

The course is devoted to graduate students and young researchers who will find, in a whole, the basic concepts of multiscale analysis and homogenization mathematical techniques in the context of periodic homogenization. The six chapters of these notes are of increasing difficulty and, each one corresponds to one day of the course.

The author would also like to thank warmly Radoslaw Wojciechowski, Toshiaki Yachimura and Lorenzo Cavallina for their very careful proofreading of the original manuscript and the many suggestions they gave to improve the presentation.

\section{Notation}

The reader is supposed to be familiar with the classical notions and notation of functional analysis. As a matter of fact, we will make frequent use the following classical notions. As usual, $\Omega$ denotes a regular open set of $\mathbb{R}^{d}$.

- Lebesgue spaces:

We will use the classical Lebesgue spaces for $1 \leq p<+\infty$

$$
L^{p}(\Omega)=\left\{f \text { measurable on } \Omega \text { such that } \int_{\Omega}|f(x)|^{p} d x<+\infty\right\} .
$$

When endowed with the norm

$$
\|f\|_{L^{p}}=\left(\int_{\Omega}|f(x)|^{p} d x\right)^{\frac{1}{p}},
$$

$L^{p}(\Omega)$ is a Banach space. For $p=2, L^{2}(\Omega)$ is a Hilbert space with the scalar product

$$
(f, g)_{L^{2}}=\int_{\Omega} f(x) g(x) d x .
$$

We will also use

$$
L^{\infty}(\Omega)=\{f \text { measurable on } \Omega \text { such that }|f(x)| \leq C \text { a.e. }\}
$$

which is a Banach space when equipped with the norm

$$
\|f\|_{L^{\infty}}=\inf \{C \text { such that } \| f(x) \mid \leq C \text { a.e. in } \Omega\} .
$$

- Distributions:

The standard language of distributions will sometimes be used. In particular we use the notation $\mathscr{D}(\Omega)$ for the space of infinitely differentiable functions with compact support in $\Omega$ and $\mathscr{D}^{\prime}(\Omega)$ for its dual, i.e. the space of distributions. Convergence in the sense of distributions (i.e. weak convergence) is also supposed to be known.

- Sobolev spaces:

Throughout these notes, we frequently use, $\Omega$ being a bounded regular domain of $\mathbb{R}^{d}$, the notation $H^{k}(\Omega)$ for the Sobolev space of degree $k \in \mathbb{N}$ defined by

$$
\begin{array}{r}
H^{k}(\Omega)=\left\{u \in L^{2}(\Omega) \text { such that } \frac{\partial^{|\alpha|} u}{\partial^{\alpha_{1}} x_{1} \cdots \partial^{\alpha_{d}} x_{d}} \in L^{2}(\Omega),\right. \\
\left.\forall \alpha=\left(\alpha_{1}, \cdots, \alpha_{d}\right) \text { s.t. }|\alpha| \leq k\right\} .
\end{array}
$$

It is well known that $H^{k}(\Omega)$ is a Hilbert space when endowed with the norm (and the associated scalar product)

$$
\|u\|_{H^{k}(\Omega)}=\left(\sum_{|\alpha| \leq k}\left\|\frac{\partial^{|\alpha|} u}{\partial^{\alpha_{1}} x_{1} \cdots \partial^{\alpha_{d}} x_{d}}\right\|_{L^{2}(\Omega)}^{2}\right)^{1 / 2} .
$$

We also use the notation 


$$
|u|_{H^{k}(\Omega)}=\left(\sum_{|\alpha|=k}\left\|\frac{\partial^{|\alpha|} u}{\partial^{\alpha_{1}} x_{1} \cdots \partial^{\alpha_{d}} x_{d}}\right\|_{L^{2}(\Omega)}^{2}\right)^{1 / 2}
$$

for the semi-norm in $H^{k}(\Omega)$.

We also use, especially for boundary value problems, $H_{0}^{1}(\Omega)$ as the closure of $\mathscr{D}(\Omega)$ under the $H^{1}$-norm.

- Hilbert spaces:

Classical Hilbert theory, especially for $L^{2}(\Omega), H^{1}(\Omega)$ and $H_{0}^{1}(\Omega)$ (but not only) will be frequently used. In particular, the reader should be familiar with the classical theory for Hilbert spaces, in particular Riesz's Theorem and Lax-Milgram Theorem. All Hilbert spaces in these notes are separable and bounded sets are weakly compact (compact for the weak convergence).

- Spaces of periodic functions:

The classical notation for a space of periodic functions consists in using a $\sharp$ subscript. In particular, for $Y=$ $(0,1)^{d}, \mathcal{C}_{\sharp}^{0}(Y)$ (resp. $\left.\mathcal{C}_{\sharp}^{k}(Y)\right)$ is the set of continuous (resp. $\left.\mathcal{C}^{k}\right)$ and $Y$-periodic functions on $\mathbb{R}^{d}$. Similarly, $H_{\sharp}^{1}(Y)$ designates the space of $H_{\mathrm{loc}}^{1}\left(\mathbb{R}^{d}\right)$ functions that are $Y$ periodic, etc.

- We will also make use of the notations $\lesssim, \gtrsim$ to indicate an inequality up to a constant. For instance $\left\|u_{\varepsilon}\right\| \lesssim\left\|v_{\varepsilon}\right\|$ means $\left\|u_{\varepsilon}\right\| \leq C\left\|v_{\varepsilon}\right\|$ where the constant $C$ is independent of $\varepsilon$.

\section{Formal Asymptotic Homogenization}

\subsection{Introduction}

Homogenization is a technical word that aims at giving a proper description of materials that are composed of several constituents, intimately mixed together. Indeed, when one considers a mixture of materials, e.g. a composite, it is expected that the new material will benefit from properties that each of its constituent only partly possess. The applications of such materials are numerous. Foam and wools are very classically used for thermic and acoustic insulation. Composed of fibers in the air or bubbles of air inside a rubber matrix, they only partly reproduce the behavior of their constituents. Other examples are given by the so-called "spring magnets" which are composed of hard and soft magnets mixed together, porous media which are a solid matrix with microchannels in which a fluid may flow or multilayer materials.

In these notes, we only consider the case of periodic homogenization for which the microstructure is periodic. Although quite restrictive at first sight, this already applies to layered materials (periodic in 1D) or tissues (2D). Moreover, the mathematical theory is very instructive. Physically, the problems may be of very different types. Elasticity for deformable bodies, fluid for porous media, or magnetic properties might be sought. The common feature among these models is that they are all described in terms of partial differential equations (PDE), the coefficients of which vary from one constituent to another, in a periodic way.

Homogenization theory is a way of seeking the averaged properties of the material from the ones of its constituents and the periodic structure as the period tends to 0 . This is a limiting process, which has very common features with weak convergence, or averaging procedures. The goal is to obtain, at the limit, a homogeneous model with homogenized coefficients that depend on the coefficients of the constituents. As we shall see, though, the procedure may not be that easy, since in some cases, the model equation may change type.

\subsection{The classical setting}

To start with we consider the simplest problem of temperature diffusion inside a body $\Omega \subset \mathbb{R}^{d}$. The equation reads

$$
\left\{\begin{array}{l}
-\operatorname{div}(A(x) \nabla u)(x)=f(x) \text { in } \Omega, \\
u=0 \text { on } \partial \Omega .
\end{array}\right.
$$

Here, $f$ is the source of heat inside the material while the tensor $A \in \mathcal{M}_{d \times d}(\mathbb{R})$ (the space of $d \times d$ real matrices) stands for the diffusion coefficients. Calling $\varepsilon$ the length of the periodic structure and $Y=(0,1)^{d}$ the unit cell that is assumed to be periodically reproduced, the presence of different materials inside $Y$ is modeled by a matrix $A(y)$ that depends on $y \in Y$. By periodicity, it is easy to extend $A$ to $\mathbb{R}^{d}$, and furthermore $A\left(\frac{x}{\varepsilon}\right)$ will represent the diffusion coefficients inside the $\varepsilon$ periodic material. Taking this into account and denoting by $u_{\varepsilon}$ the solution on the periodically microstructured material, we transform the preceding problem into

$$
\left\{\begin{array}{l}
-\operatorname{div}\left(A\left(\frac{x}{\varepsilon}\right) \nabla u_{\varepsilon}\right)(x)=f(x) \text { in } \Omega, \\
u_{\varepsilon}=0 \text { on } \partial \Omega .
\end{array}\right.
$$

The main question for the homogenization procedure consists in finding possible limit(s) $u_{0}$ to the sequence $\left(u_{\varepsilon}\right)_{\varepsilon>0}$ and identifying the problem(s) that $u_{0}$ solves. 

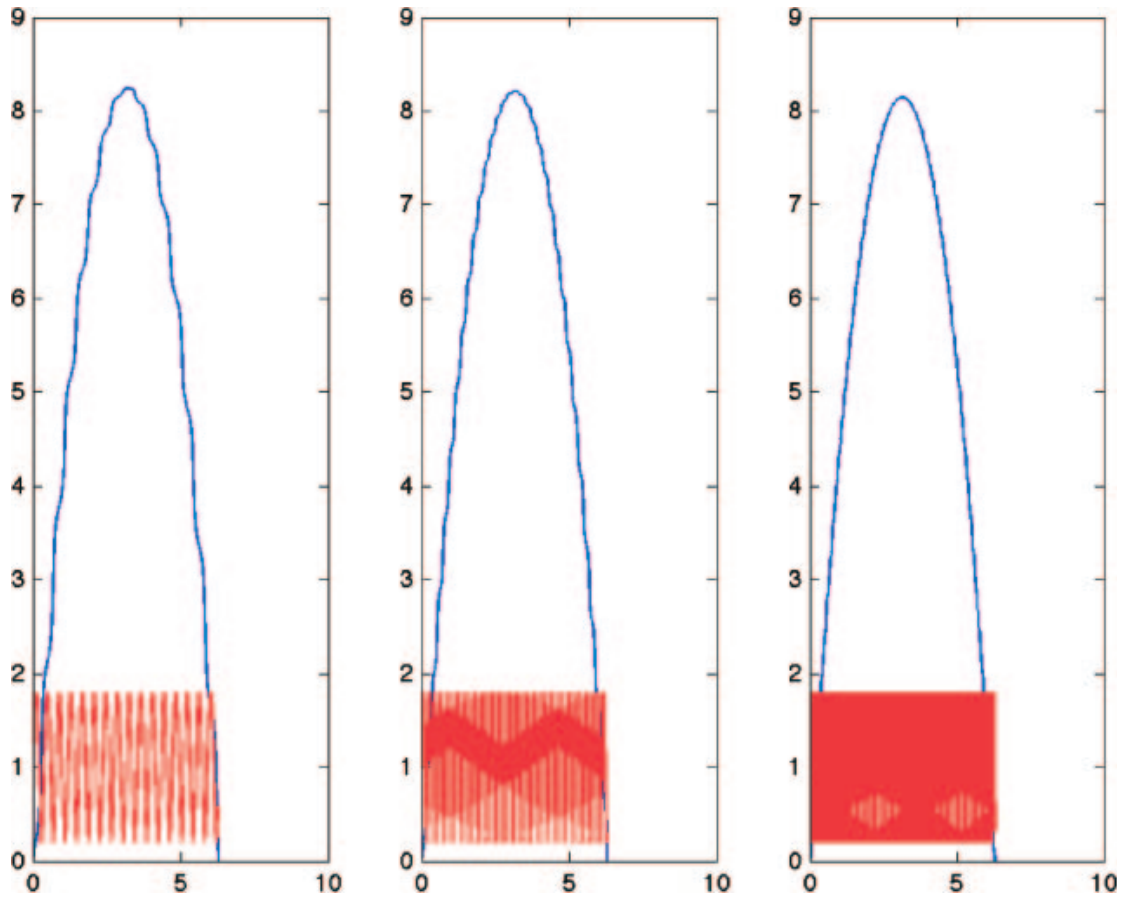

Fig. 1.1. The solution to the boundary value problem (1.3) computed for $f=1$ and three values $\varepsilon=0.01$ (left), $\varepsilon=0.005$ (middle) and $\varepsilon=0.00025$ (right). It shows an oscillation that decays with $\varepsilon$ at a frequency that increases with $\varepsilon$. The graph of $A$ is shown in red.

Notice that under classical assumptions on the tensor $A$, namely the existence of $0<c<C$ such that

$$
\forall \xi \in \mathbb{R}^{d}, c|\xi|^{2} \leq(A \xi, \xi) \leq C|\xi|^{2}
$$

and the fact that $f \in L^{2}(\Omega)$, the problem (1.1) possesses a unique solution $u_{\varepsilon} \in H_{0}^{1}(\Omega)$ that furthermore satisfies

$$
\begin{aligned}
\int_{\Omega}\left(A\left(\frac{x}{\varepsilon}\right) \nabla u_{\varepsilon}, \nabla u_{\varepsilon}\right) d x & =\int_{\Omega} f(x) u_{\varepsilon}(x) d x \\
& \leq\|f\|_{L^{2}}\left\|u_{\varepsilon}\right\|_{L^{2}} \\
& \leq C_{P}\|f\|_{L^{2}}\left\|\nabla u_{\varepsilon}\right\|_{L^{2}}
\end{aligned}
$$

where $C_{P}$ stands for the Poincaré constant of $\Omega$. Using the coerciveness assumption (1.2), one easily deduces

$$
\begin{aligned}
\left\|\nabla u_{\varepsilon}\right\|_{L^{2}} & \leq \frac{C_{P}}{c}\|f\|_{L^{2}} \\
& \lesssim\|f\|_{L^{2}}
\end{aligned}
$$

and therefore the sequence $\left(u_{\varepsilon}\right)_{\varepsilon>0}$ is uniformly bounded in $H_{0}^{1}(\Omega)$.

As an example we consider the problem in 1D

$$
\left\{\begin{array}{l}
-\left(A\left(\frac{x}{\varepsilon}\right) u_{\varepsilon}^{\prime}\right)^{\prime}(x)=1 \text { on }(0,1), \\
u_{\varepsilon}(0)=u_{\varepsilon}(1)=0
\end{array}\right.
$$

where $A(y)=1+0.8 \sin (2 \pi y)$. The solution $u_{\varepsilon}$, computed with a finite element code is plotted in Fig. 1.1 for three values of $\varepsilon$.

\subsection{Multiscale expansion}

The multiscale expansion method is a heuristic that finds the correct behavior of the sequence $\left(u_{\varepsilon}\right)_{\varepsilon>0}$. It consists of assuming the multiscale expansion

$$
u_{\varepsilon}(x)=u_{0}(x, x / \varepsilon)+\varepsilon u_{1}(x, x / \varepsilon)+\varepsilon^{2} u_{2}(x, x / \varepsilon)+\cdots,
$$

where $u_{i}(x, y)$ are assumed to be periodic in the $y \in Y$ variable, plugging this ansatz into the equation, and equating all terms of the same orders in powers of $\varepsilon$. Notice that each term of the expansion depends on both the slow variable $x$ and the fast variable $y=x / \varepsilon$. Notice also that when computing gradients on $u_{i}(x, x / \varepsilon)$ one obtains 


$$
\nabla\left[u_{i}(x, x / \varepsilon)\right]=\left(\nabla_{x} u_{i}\right)(x, x / \varepsilon)+\frac{1}{\varepsilon}\left(\nabla_{y} u_{i}\right)(x, x / \varepsilon) .
$$

Therefore the expansion (1.4) leads to the following equations

- Order $\varepsilon^{-2}$ :

$$
-\operatorname{div}_{y}\left(A(y) \nabla_{y} u_{0}(x, y)\right)=0
$$

- $\operatorname{Order} \varepsilon^{-1}$ :

$$
-\operatorname{div}_{y}\left(A(y)\left(\nabla_{x} u_{0}+\nabla_{y} u_{1}\right)\right)(x, y)-\operatorname{div}_{x}\left(A(y) \nabla_{y} u_{0}\right)(x, y)=0,
$$

- Order $\varepsilon^{0}$ :

$$
-\operatorname{div}_{x}\left(A(y)\left(\nabla_{x} u_{0}+\nabla_{y} u_{1}\right)\right)-\operatorname{div}_{y}\left(A(y)\left(\nabla_{x} u_{1}+\nabla_{y} u_{2}\right)\right)=f(x),
$$

Remark 1.1. Notice that in deriving the preceding equations we have assumed that they were valid for any $y \in Y$ and not only for $y=x / \varepsilon$.

We now proceed step by step to solve the preceding system of equations.

- Order $\varepsilon^{-2}$ : Multiplying the Eq. (1.5) by $u_{0}$ and integrating by parts over $Y$ leads to

$$
\int_{Y}\left(A(y) \nabla_{y} u_{0}, \nabla_{y} u_{0}\right)(x, y) d y=0
$$

which, in view of the uniform coerciveness of $A(1.2)$, leads to $\nabla_{y} u_{0}(x, y)=0$ and the fact that

$$
u_{0}(x, y)=u_{0}(x)
$$

does not depend on the $y$ variable.

- Order $\varepsilon^{-1}$ : Since $u_{0}$ does not depend on $y$, the Eq. (1.6) simplifies to

$$
-\operatorname{div}_{y}\left(A(y)\left(\nabla_{x} u_{0}+\nabla_{y} u_{1}\right)\right)(x, y)=0 .
$$

We take the $x$ variable as a parameter, and notice that $\nabla_{x} u_{0}(x)=\sum_{i=1}^{d} \frac{\partial u_{0}}{\partial x_{i}}(x) e_{i}$ where $e_{i}$ is the $i$-th basis vector of $\mathbb{R}^{d}$. We also call $\omega_{i}(y) \in H_{\sharp}^{1}(Y)$ the (unique up to an additive constant) solution to

$$
\left.-\operatorname{div}_{y}\left(A(y) \nabla_{y} \omega_{i}\right)\right)(y)=\operatorname{div}_{y}\left(A(y) e_{i}\right)
$$

and deduce by linearity that

$$
u_{1}(x, y)=\sum_{i=1}^{d} \frac{\partial u_{0}}{\partial x_{i}}(x) \omega_{i}(y)
$$

- Order $\varepsilon^{0}$ : We remark that, up to now, we have not been able to solve the problem, but we have rather found a constraint that $u_{0}$ needs to solve and expressed $u_{1}$ in terms of $u_{0}$. It seems hopeless that this new equation will close the system since a new variable, namely $u_{2}$ has been introduced. We will see that actually we will cancel the unknown $u_{2}$ and that only a closed system remains. Namely, integrating ${ }^{1}$ Eq. (1.7) over $y \in Y$ and making use of the periodicity of $u_{1}$ and $u_{2}$ leads to

$$
-\operatorname{div}_{x} \int_{Y}\left(A(y)\left(\nabla_{x} u_{0}+\nabla_{y} u_{1}\right)\right)(x, y) d y=\int_{Y} f(x) d y=f(x) .
$$

The unknown $u_{2}$ has disappeared, and we are left with another equation coupling $u_{0}$ and $u_{1}$, that we can solve. Indeed, using (1.10), we infer

$$
\nabla_{y} u_{1}(x, y)=\sum_{i=1}^{d} \frac{\partial u_{0}}{\partial x_{i}}(x) \nabla_{y} \omega_{i}(y)
$$

and (1.11) becomes

$$
-\operatorname{div}_{x} \int_{Y}\left(A(y)\left(\nabla_{x} u_{0}+\sum_{i=1}^{d} \frac{\partial u_{0}}{\partial x_{i}}(x) \nabla_{y} \omega_{i}(y)\right)\right)(x, y) d y=f(x) .
$$

This latter equation may be rewritten as

$$
-\operatorname{div}_{x}\left(A_{\mathrm{eff}} \nabla_{x} u_{0}\right)=f(x)
$$

\footnotetext{
${ }^{1}$ Actually, for this equation to have a solution in $u_{2}$, the right-hand side needs to be null averaged. Therefore, we not only made $u_{2}$ disappear, but we write a necessary and sufficient condition for this equation to have a solution $u_{2}$. This is sometimes called in this context the "Fredholm's alternative" although actually this is only a particular case of this much more general concept. See Exercise 1.3 for more details about this.
} 
where the effective diffusion tensor $A_{\text {eff }}$ is explicitly given by

$$
A_{\mathrm{eff}, j k}=\int_{Y}\left(A_{j k}(y)+\sum_{l=1}^{d} A_{j l} \frac{\partial \omega_{k}}{\partial y_{l}}(y)\right) d y
$$

It remains to find the boundary conditions that $u_{0}$ needs to fulfill. Here, since we simply deal with a Dirichlet boundary condition $u_{\varepsilon}=0$ on $\partial \Omega$, the multiscale expansion gives at order $\varepsilon^{0}$ :

$$
u_{0}(x, y)=u_{0}(x)=0 \text { on } \partial \Omega .
$$

Therefore, the limit $u_{0}$ satisfies the boundary value problem

$$
\left\{\begin{array}{l}
-\operatorname{div}_{x}\left(A_{\text {eff }} \nabla_{x} u_{0}\right)=f(x) \text { in } \Omega, \\
u_{0}=0 \text { on } \partial \Omega
\end{array}\right.
$$

Remark 1.2. Equation (1.9) permits us to solve $u_{1}$ in terms of $u_{0}$. It is usually called the cell problem as it holds on the unit cell $Y$. Similarly, Eq. (1.11) gives the equation solved by $u_{0}$ (the limiting solution in the multiscale expansion) and is called the homogenized problem. The functions $\omega_{i}$ that appear in the cell problem (1.9) are usually called the correctors. It is also noteworthy to remark that the problem (1.9) is of the form

$$
\left\{\begin{array}{l}
-\operatorname{div}\left(A(y) \nabla_{y} \omega\right)=f \\
\omega \text { is } Y \text {-periodic. }
\end{array}\right.
$$

This latter problem possesses a unique solution up to an additive constant if and only if the compatibility condition

$$
\int_{Y} f(y) d y=0
$$

holds (see Exercise 1.3). This property, sometimes called Fredholm's alternative was also used to hope for a solution $u_{2}$ of (1.7), although we are not interested in this solution.

Remark 1.3. In the homogenized problem, the diffusion tensor $A_{\text {eff }}$ does not depend on $x$. It only depends on the unit cell distribution of the diffusion tensor $A$ (or equivalently the materials involved) and the resolution of the cell problems.

\subsection{The cell and the homogenized problems}

Although not rigorous, the multiscale expansion problem enabled us to give the limit $u_{0}$ of $u_{\varepsilon}$ as $\varepsilon$ tends to 0 . It is expressed as the solution of a boundary value problem that involves the cell problem. However, a few questions remain to be examined:

- Is the homogenized problem well-posed? Can we say something about the coerciveness of $A_{\mathrm{eff}}$ ?

- Is the cell problem well-posed? In particular, the fact that the correctors $\omega_{i}$ are assumed to be $Y$-periodic may create an artificial constraint.

- What do these correctors in the initial problem mean?

We will look at those questions one after the other, and actually in the reverse order. To start with, we use the formula (1.10) that expresses $u_{1}$ in terms of $u_{0}$ and the correctors

$$
u_{1}(x, y)=\sum_{i=1}^{d} \frac{\partial u_{0}}{\partial x_{i}}(x) \omega_{i}(y)
$$

and use it in the multiscale expression for $u_{\varepsilon}$. We obtain

$$
\begin{aligned}
u_{\varepsilon}(x) & \sim u_{0}(x)+\varepsilon u_{1}\left(x, \frac{x}{\varepsilon}\right) \\
& \sim u_{0}(x)+\varepsilon \sum_{i=1}^{d} \frac{\partial u_{0}}{\partial x_{i}}(x) \omega_{i}\left(\frac{x}{\varepsilon}\right) .
\end{aligned}
$$

We thus see that the solution $u_{\varepsilon}$ oscillates with an amplitude $\varepsilon$ and with a profile $\omega_{i}$ scaled by $\frac{\partial u_{0}}{\partial x_{i}}(x)$. This in particular explains why in Fig. 1.1 the solution oscillates less and less where $u_{0}$ has its maximum.

Let us now turn to the cell problem (1.9), that we recall hereafter:

$$
-\operatorname{div}_{y}\left(A(y) \nabla_{y} \omega_{i}\right)(y)=\operatorname{div}_{y}\left(A(y) e_{i}\right)
$$

The associated variational formulation of the problem is obtained by multiplying the equation by $\phi \in H_{\sharp}^{1}(Y)$ and integrating by parts. We obtain 


$$
\int_{Y}\left(A(y) \nabla_{y} \omega_{i}(y), \nabla_{y} \phi(y)\right) d y=-\int_{Y}\left(A(y) e_{i}, \nabla_{y} \phi(y)\right) d y,
$$

whose existence and uniqueness of the solution follows from the Lax-Milgram Theorem in the Hilbert space

$$
V=\left\{\psi \in H_{\sharp}^{1}(Y), \text { s.t. } \int_{Y} \psi(y) d y=0\right\}
$$

using the coerciveness assumption (1.2). (See Exercises 1.2 and 1.3.) Notice that testing with $\phi=\omega_{k}$ leads to

$$
\int_{Y}\left(A(y)\left(e_{i}+\nabla_{y} \omega_{i}(y)\right), \nabla_{y} \omega_{k}(y)\right) d y=0,
$$

from which we deduce that the homogenized effective tensor $A_{\text {eff }}$, defined by (1.14), satisfies

$$
\begin{aligned}
A_{\mathrm{eff}, j k} & =\int_{Y}\left(A_{j k}(y)+\sum_{l=1}^{d} A_{j l}(y) \frac{\partial \omega_{k}}{\partial y_{l}}(y)\right) d y \\
& =\int_{Y}\left(A(y) e_{k}+A(y) \nabla_{y} \omega_{k}(y), e_{j}\right) d y \\
& =\int_{Y}\left(A(y)\left(e_{k}+\nabla_{y} \omega_{k}(y)\right), e_{j}\right) d y \\
& =\int_{Y}\left(A(y)\left(e_{k}+\nabla_{y} \omega_{k}(y)\right), e_{j}+\nabla_{y} \omega_{j}\right) d y
\end{aligned}
$$

because of (1.17). Therefore, if $\xi \in \mathbb{R}^{d}$, then

$$
\begin{aligned}
\left(A_{\mathrm{eff}} \xi, \xi\right) & =\sum_{j, k=1}^{d} A_{\mathrm{eff}, j k} \xi_{j} \xi_{k} \\
& =\int_{Y}\left(A(y)\left(\xi+\sum_{k=1}^{d} \xi_{k} \nabla_{y} \omega_{k}(y)\right), \xi+\sum_{j=1}^{d} \xi_{j} \nabla_{y} \omega_{j}\right) d y \\
& \gtrsim\left\|\xi+\sum_{k=1}^{d} \xi_{k} \nabla_{y} \omega_{k}(y)\right\|_{L^{2}(Y)}^{2}
\end{aligned}
$$

which shows that $A_{\text {eff }}$ is a positive definite matrix. Considering the homogenized problem

$$
\left\{\begin{array}{l}
-\operatorname{div}_{x}\left(A_{\text {eff }} \nabla_{x} u_{0}\right)=f(x) \text { in } \Omega, \\
u_{0}=0 \text { on } \partial \Omega,
\end{array}\right.
$$

where the homogenized tensor $A_{\text {eff }}$ is given by (1.14), we obtain that the classical theory of elliptic problems applies and that it possesses a unique solution, again thanks to the Lax-Milgram Theorem.

\subsection{Exercises}

Exercise 1.1. Homogenized problem in 1D.

We here consider the model problem (1.1) in 1D

$$
\left\{\begin{array}{l}
-\left(A\left(\frac{x}{\varepsilon}\right) u_{\varepsilon}^{\prime}\right)^{\prime}(x)=f(x) \text { on }(0,1), \\
u_{\varepsilon}(0)=u_{\varepsilon}(1)=0
\end{array}\right.
$$

where $f \in L^{2}(0,1)$ and $A$ is a 1-periodic function that satisfies

$$
\exists C>c>0, \forall y \in(0,1), c \leq A(y) \leq C .
$$

Show that the homogenized equation associated to the problem is given by

$$
\left\{\begin{array}{l}
-\left(A_{\mathrm{eff}} u_{0}^{\prime}\right)^{\prime}(x)=f(x) \text { on }(0,1) \\
u_{0}(0)=u_{0}(1)=0
\end{array}\right.
$$

where

$$
A_{\text {eff }}=\left(\int_{0}^{1} \frac{d y}{A(y)}\right)^{-1}
$$


Exercise 1.2. The cell problem.

Let $V=\left\{\psi \in H_{\sharp}^{1}(Y)\right.$ s.t. $\left.\int_{Y} \psi(y) d y=0\right\}$ the Hilbert space endowed with the norm (and associated scalar product)

$$
\|\psi\|_{V}=\left(\int_{Y}\left|\nabla_{y} \psi(y)\right|^{2} d y\right)^{\frac{1}{2}} .
$$

Show, using the Lax-Milgram Theorem, that the variational formulation of the cell problem

Find $\omega_{i} \in V$ such that $\forall \phi \in V$,

$$
\int_{Y}\left(A(y) \nabla_{y} \omega_{i}(y), \nabla_{y} \phi(y)\right) d y=-\int_{Y}\left(A(y) e_{i}, \nabla_{y} \phi(y)\right) d y,
$$

where $A$ satisfies the coerciveness assumption (1.2) possesses a unique solution.

Exercise 1.3. Fredholm alternative.

Let $V=\left\{\psi \in H_{\sharp}^{1}(Y)\right.$, s.t. $\left.\int_{Y} \psi(y) d y=0\right\}$ the Hilbert space endowed with the norm (and associated scalar product)

$$
\|\psi\|_{V}=\left(\int_{Y}\left|\nabla_{y} \psi(y)\right|^{2} d y\right)^{\frac{1}{2}}
$$

and $f \in L^{2}(Y)$.

1. Show, using the Lax-Milgram Theorem, that the variational formulation of the cell problem

$$
\text { Find } \omega_{i} \in V \text { such that } \forall \phi \in V, \int_{Y}\left(A(y) \nabla_{y} \omega_{i}(y), \nabla_{y} \phi(y)\right) d y=-\int_{Y}\left(A(y) e_{i}, \nabla_{y} \phi(y)\right) d y \text {, }
$$

where $A$ satisfies the coerciveness assumption (1.2) possesses a unique solution.

2. Show that it is also a solution to

$$
-\operatorname{div}\left(A(y) \nabla_{y} \omega\right)=f
$$

under the necessary and sufficient condition that

$$
\int_{Y} f(y) d y=0
$$

Exercise 1.4. Extend all the preceding multiscale analysis to the case where the diffusion tensor $A$ depends also on the slow variable $x$, namely $A:=A(x, y)$.

\section{Two-scale Convergence}

\subsection{Introduction}

As we have seen in the preceding chapter, the multiscale expansion method answers questions concerning the behavior and the limit solution of the homogenization problem. However, the method that we have developed is unsatisfactory for two reasons:

- First, it is heuristic. We have only postulated an ansatz and somehow shown that the limit solution $u_{0}$ should satisfy the homogenized problem (1.11). At this stage, we have no clue about the fact that this ansatz is true, and no proof about any convergence of the sequence $\left(u_{\varepsilon}\right)_{\varepsilon}$ as $\varepsilon$ tends to 0 .

- The approach that we have used works in two steps. We have postulated the ansatz, and obtained the set of equations that the limit should satisfy (the cell and homogenized problems). Then, we have proven the existence and uniqueness of the solution to these problems. It would be more convenient to get, as a whole, the problems and the limit.

The 2-scale convergence method is exactly intended to overcome both of these issues. As we shall see, it provides the user with a rigorous theoretical framework that enables him or her to work out directly the problems (both the cell and homogenized problems) and prove the convergence of the family $\left(u_{\varepsilon}\right)_{\varepsilon}$ in a suitable sense.

The following discussion is based on the theory proposed by Nguetseng [16] and further developed by Allaire [1].

\subsection{Two-scale convergence}

The basic notion that one needs to introduce is the following.

Definition 2.1. A sequence $\left(u_{\varepsilon}\right)_{\varepsilon>0}$ in $L^{2}(\Omega)$ is said to two-scale converge to a limit $u_{0}(x, y) \in L^{2}(\Omega \times Y)$ if, for any $\psi(x, y) \in \mathscr{D}\left(\Omega ; \mathcal{C}_{\sharp}^{\infty}(Y)\right)$ we have

$$
\lim _{\varepsilon \rightarrow 0} \int_{\Omega} u_{\varepsilon}(x) \psi\left(x, \frac{x}{\varepsilon}\right) d x=\int_{\Omega \times Y} u_{0}(x, y) \psi(x, y) d x d y .
$$


In this case we denote the two-scale convergence by

$$
u_{\varepsilon} \rightarrow u_{0} \text { two-scale as } \varepsilon \rightarrow 0 .
$$

The main reason for introducing the two-scale convergence is the following compactness theorem (we refer the reader to $[1,16]$ for the proof).

Theorem 2.1. Let $\left(u_{\varepsilon}\right)_{\varepsilon>0}$ be a sequence bounded in $L^{2}(\Omega)$. There exists a subsequence $\left(u_{\varepsilon_{n}}\right)_{n \in \mathbb{N}}$ and $u_{0} \in L^{2}(\Omega \times Y)$ such that

$$
\lim _{n \rightarrow \infty} \varepsilon_{n}=0, \text { and } u_{\varepsilon_{n}} \rightarrow u_{0} \text { two-scale as } n \rightarrow+\infty \text {. }
$$

As we shall see, the preceding compactness theorem generalizes the well known compactness theorem in $L^{2}(\Omega)$ (from a bounded sequence in $L^{2}(\Omega)$ one can extract a subsequence that weakly converges in $L^{2}(\Omega)$ ). This is not surprising since the definition of two-scale convergence is written under a weak form. It is a convergence for any test function and not convergence in norm.

\subsection{Admissible functions}

We will make frequent use of the fact that for $\psi \in \mathcal{D}\left(\Omega, \mathcal{C}_{\sharp}(Y)\right)$ one has

$$
\lim _{\varepsilon \rightarrow 0} \int_{\Omega} \psi\left(x, \frac{x}{\varepsilon}\right)^{2} d x=\int_{\Omega \times Y} \psi(x, y)^{2} d x d y .
$$

(See Exercise 2.2.) It is however unclear whether the regularity of $\psi$ may be weakened. We therefore introduce the following definition.

Definition 2.2. A function $\psi$ that satisfies (2.2) is called admissible.

It turns out that this is a subtle notion. Indeed, for a given function $\psi \in L^{2}(\Omega \times Y)$ there is no reason for the function

$$
x \mapsto \psi\left(x, \frac{x}{\varepsilon}\right)
$$

to be even measurable. The complete space of admissible functions is not known much more precisely, it is however known that any Caratheory function (continuous in one variable and measurable in the other) is admissible. Therefore, functions in $L^{p}\left(\Omega, \mathcal{C}_{\sharp}(Y)\right)$ as well as $L_{\sharp}^{p}(Y, \mathcal{C}(\Omega))$ are admissible. We also refer to [1] for an explicit construction of a non admissible function which belongs to $\mathcal{C}\left(\Omega, L_{\sharp}^{1}(Y)\right)$.

\subsection{Properties}

The main property of two-scale convergence is the convergence of norms.

Proposition 2.1. Let $\left(u_{\varepsilon}\right)_{\varepsilon>0}$ be a sequence in $L^{2}(\Omega)$ that two-scale converges to $u_{0} \in L^{2}(\Omega \times Y)$. Then

$$
\begin{gathered}
u_{\varepsilon} \rightarrow u(x)=\int_{Y} u_{0}(x, y) d y \text { weakly in } L^{2}(\Omega), \\
\underset{\varepsilon \rightarrow 0}{\liminf }\left\|u_{\varepsilon}\right\|_{L^{2}} \geq\left\|u_{0}\right\|_{L^{2}(\Omega \times Y)} \geq\|u\|_{L^{2}(\Omega)} .
\end{gathered}
$$

Proof. We first remark that taking a test function $\psi(x, y)=\psi(x)$ that does not depend on the $y$ variable in the definition of the two-scale convergence immediately gives (2.3). In order to prove (2.4), we consider $\psi \in L^{2}\left(\Omega, \mathcal{C}_{\sharp}(Y)\right)$, and expand

$$
\begin{aligned}
\int_{\Omega}\left(u_{\varepsilon}(x)-\psi\left(x, \frac{x}{\varepsilon}\right)\right)^{2} d x= & \int_{\Omega}\left(u_{\varepsilon}(x)\right)^{2} d x-2 \int_{\Omega} u_{\varepsilon}(x) \psi\left(x, \frac{x}{\varepsilon}\right) d x \\
& +\int_{\Omega}\left(\psi\left(x, \frac{x}{\varepsilon}\right)\right)^{2} d x \\
\geq & 0 .
\end{aligned}
$$

Passing to the liminf, using the definition of two-scale convergence, we obtain

$$
\liminf _{\varepsilon \rightarrow 0} \int_{\Omega}\left(u_{\varepsilon}(x)\right)^{2} d x \geq 2 \int_{\Omega \times Y} u_{0}(x, y) \psi(x, y) d x d y-\int_{\Omega \times Y} \psi(x, y)^{2} d x d y
$$

since $\psi$ is admissible.

Using this inequality for a sequence of smooth functions $\left(\psi_{n}\right)_{n \in \mathbb{N}}$ that converges to $u_{0}$ in $L^{2}(\Omega \times Y)$ leads to 


$$
\liminf _{\varepsilon \rightarrow 0} \int_{\Omega}\left(u_{\varepsilon}(x)\right)^{2} d x \geq \int_{\Omega \times Y} u_{0}(x, y)^{2} d x d y .
$$

Using Cauchy-Schwarz inequality immediately leads to the second inequality in (2.4).

As for $L^{2}$ convergence, we also have the strong version of the preceding proposition.

Proposition 2.2. Let $\left(u_{\varepsilon}\right)_{\varepsilon>0}$ be a sequence in $L^{2}(\Omega)$ that two-scale converges to $u_{0} \in L^{2}(\Omega \times Y)$ and is such that

$$
\lim _{\varepsilon \rightarrow 0}\left\|u_{\varepsilon}\right\|_{L^{2}}=\left\|u_{0}\right\|_{L^{2}(\Omega \times Y)} .
$$

Then, for any sequence $\left(v_{\varepsilon}\right)_{\varepsilon>0}$ in $L^{2}(\Omega)$ that two-scale converges to $v_{0} \in L^{2}(\Omega \times Y)$, one has

$$
u_{\varepsilon} v_{\varepsilon} \rightarrow \int_{Y} u_{0}(x, y) v_{0}(x, y) d y \text { in } \mathscr{D}^{\prime}(\Omega)
$$

and if $u_{0} \in L^{2}\left(\Omega, \mathcal{C}_{\sharp}(Y)\right)$

$$
\lim _{\varepsilon \rightarrow 0}\left\|u_{\varepsilon}(x)-u_{0}\left(x, \frac{x}{\varepsilon}\right)\right\|_{L^{2}}=0
$$

Proof. The proof follows readily the same lines as before. We take a sequence of smooth function $\psi_{n} \in L^{2}\left(\Omega, \mathcal{C}_{\sharp}(Y)\right)$ that converges to $u_{0}(x, y)$ in $L^{2}(\Omega \times Y)$. We have with the definition of two-scale convergence and the fact that $\psi_{n}$ is smooth

$$
\lim _{\varepsilon \rightarrow 0} \int_{\Omega}\left(u_{\varepsilon}(x)-\psi_{n}\left(x, \frac{x}{\varepsilon}\right)\right)^{2} d x=\int_{\Omega \times Y}\left(u_{0}(x, y)-\psi_{n}(x, y)\right)^{2} d x d y
$$

and therefore

$$
\lim _{n \rightarrow+\infty} \lim _{\varepsilon \rightarrow 0} \int_{\Omega}\left(u_{\varepsilon}(x)-\psi_{n}\left(x, \frac{x}{\varepsilon}\right)\right)^{2} d x=0
$$

Now, for any $\phi \in \mathscr{D}(\Omega)$, one has

$$
\begin{aligned}
\int_{\Omega} u_{\varepsilon}(x) v_{\varepsilon}(x) \phi(x) d x= & \int_{\Omega} \psi_{n}\left(x, \frac{x}{\varepsilon}\right) v_{\varepsilon}(x) \phi(x) d x \\
& +\int_{\Omega}\left(u_{\varepsilon}(x)-\psi_{n}\left(x, \frac{x}{\varepsilon}\right)\right) v_{\varepsilon}(x) \phi(x) d x .
\end{aligned}
$$

We now use the fact that $\left(v_{\varepsilon}\right)_{\varepsilon}$ is bounded and the preceding result to deduce, passing to the limit $\varepsilon \rightarrow 0$

$$
\limsup _{\varepsilon \rightarrow 0}\left|\int_{\Omega} u_{\varepsilon}(x) v_{\varepsilon}(x) \phi(x) d x-\int_{\Omega \times Y} \phi(x) \psi_{n}(x, y) v_{0}(x, y) d x d y\right| \leq C\left\|u_{0}-\psi_{n}\right\|_{L^{2}(\Omega \times Y)} .
$$

Passing now to the limit $n \rightarrow+\infty$ gives the result

$$
\lim _{\varepsilon \rightarrow 0} \int_{\Omega} u_{\varepsilon}(x) v_{\varepsilon}(x) \phi(x) d x=\int_{\Omega \times Y} \phi(x) u_{0}(x, y) v_{0}(x, y) d x d y,
$$

which is nothing but (2.6). If $u_{0}$ is smooth enough (this would be the case for instance if $u_{0} \in L^{2}\left(\Omega, \mathcal{C}_{\sharp}(Y)\right)$ ), then one can take $\psi_{n}=u_{0}$ in the beginning of the proof to obtain (2.7).

Up to now, we have given the main results about $L^{2}$ bounded sequences. For sequences bounded in $H^{1}(\Omega)$, the results can be made more precise.

Theorem 2.2. Let $\left(u_{\varepsilon}\right)_{\varepsilon>0}$ be a sequence bounded in $H^{1}(\Omega)$. Then there exist $u_{0} \in H^{1}(\Omega)$ and $u_{1} \in L^{2}\left(\Omega, H_{\sharp}^{1}(Y) / \mathbb{R}\right)$ such that, up to the extraction of a subsequence, one has

$$
\begin{aligned}
& u_{\varepsilon} \rightarrow u_{0} \text { weakly in } H^{1}(\Omega), \\
& u_{\varepsilon} \rightarrow u_{0} \text { strongly in } L^{2}(\Omega), \\
& u_{\varepsilon} \rightarrow u_{0} \text { two-scale, } \\
& \nabla u_{\varepsilon} \rightarrow \nabla_{x} u_{0}(x)+\nabla_{y} u_{1}(x, y) \text { two-scale. }
\end{aligned}
$$

Proof. The first two statements are well-known properties of convergence in $H^{1}(\Omega)$ and the Rellich Theorem. We also infer, from the boundedness of $\left(u_{\varepsilon}\right)_{\varepsilon>0}$ and $\left(\nabla u_{\varepsilon}\right)_{\varepsilon>0}$, the existence of $U(x, y) \in L^{2}(\Omega \times Y)$ and $\xi(x, y) \in L^{2}(\Omega \times Y)^{d}$ such that (up to a subsequence) 


$$
\begin{aligned}
& u_{\varepsilon} \rightarrow U \text { two-scale, } \\
& \nabla u_{\varepsilon} \rightarrow \xi(x, y) \text { two-scale. }
\end{aligned}
$$

This means that for any test functions $\psi \in \mathscr{D}\left(\Omega, \mathcal{C}_{\sharp}(Y)\right)$ and $\Psi \in \mathscr{D}\left(\Omega, \mathcal{C}_{\sharp}(Y)\right)^{d}$

$$
\begin{aligned}
& \int_{\Omega} u_{\varepsilon}(x) \psi\left(x, \frac{x}{\varepsilon}\right) d x \rightarrow \int_{\Omega \times Y} U(x, y) \psi(x, y) d x d y, \\
& \int_{\Omega} \nabla u_{\varepsilon}(x) \cdot \Psi\left(x, \frac{x}{\varepsilon}\right) d x \rightarrow \int_{\Omega \times Y} \xi(x, y) \cdot \Psi(x, y) d x d y .
\end{aligned}
$$

But, an integration by parts shows that

$$
\varepsilon \int_{\Omega} \nabla u_{\varepsilon}(x) \cdot \Psi\left(x, \frac{x}{\varepsilon}\right) d x=-\int_{\Omega} u_{\varepsilon}(x)\left(\operatorname{div}_{y} \Psi\left(x, \frac{x}{\varepsilon}\right)+\varepsilon \operatorname{div}_{x} \Psi\left(x, \frac{x}{\varepsilon}\right)\right) d x,
$$

which gives, passing to the limit $\varepsilon \rightarrow 0$

$$
0=-\int_{\Omega \times Y} U(x, y) \operatorname{div}_{y} \Psi(x, y) d x d y
$$

or equivalently that $U(x, y)$ does not depend on $y$. Therefore $U(x, y)=U(x)$ and $u_{0}(x)=\int_{Y} U(x, y) d y=U(x)$. This shows (2.10). In order to show (2.11), we take a test function $\Psi$ such that $\operatorname{div}_{y} \Psi(x, y)=0$. We get

$$
\int_{\Omega} \nabla u_{\varepsilon}(x) \cdot \Psi\left(x, \frac{x}{\varepsilon}\right) d x=-\int_{\Omega} u_{\varepsilon}(x) \operatorname{div}_{x} \Psi\left(x, \frac{x}{\varepsilon}\right) d x
$$

which, passing to the limit, leads to

$$
\begin{aligned}
\int_{\Omega \times Y} \xi(x, y) \cdot \Psi(x, y) d x d y & =-\int_{\Omega \times Y} u_{0}(x) \operatorname{div}_{x} \Psi(x, y) d x d y \\
& =\int_{\Omega} \nabla_{x} u_{0}(x) \cdot\left(\int_{Y} \Psi(x, y) d y\right) d x .
\end{aligned}
$$

Thus, for any $\Psi \in \mathscr{D}\left(\Omega, \mathcal{C}_{\sharp}^{\infty}(Y)\right)^{d}$ such that $\operatorname{div}_{y} \Psi=0$, one has

$$
\int_{\Omega \times Y}\left(\xi(x, y)-\nabla_{x} u_{0}(x)\right) \cdot \Psi(x, y) d x d y=0 .
$$

This is sufficient to deduce that there exists $u_{1} \in L^{2}\left(\Omega, H_{\sharp}^{1}(Y) / \mathbb{R}\right)$ such that

$$
\xi(x, y)=\nabla_{x} u_{0}(x)+\nabla_{y} u_{1}(x, y)
$$

(see Exercise 2.5)

\subsection{Exercises}

Exercise 2.1. Admissible functions.

1. Show that a continuous function $f \in \mathcal{C}\left(\Omega, \mathcal{C}_{\sharp}(Y)\right)$ is admissible in the sense of Definition 2.2.

Hint: Since $f$ in continuous, one has $\forall \delta>0, \exists \varepsilon_{0}>0$ such that $\forall \varepsilon<\varepsilon_{0}$,

$$
\left|x-x^{\prime}\right| \leq \varepsilon \Rightarrow\left\|f(x, \cdot)-f\left(x^{\prime}, \cdot\right)\right\|_{L^{\infty}(Y)} \leq \delta .
$$

Therefore, to within an arbitrarily small error $\delta$, one can approximate $f\left(x, \frac{x}{\varepsilon}\right)$ by $f\left(x_{i}, \frac{x}{\varepsilon}\right)$ on the cube $\varepsilon(i+Y)$ where $i \in \mathbb{Z}^{d}$ and with $x_{i} \in \varepsilon(i+Y)$.

2. Show that a function $\psi(x, y)=\theta(x) \eta(y)$ where $\theta \in L^{2}(\Omega)$ and $\eta \in L_{\sharp}^{2}(Y)$ is admissible in the sense of Definition 2.2.

3. Let $f$ and $g$ be two admissible functions. Show that

$$
\lim _{\varepsilon \rightarrow 0} \int_{\Omega} f\left(x, \frac{x}{\varepsilon}\right) g\left(x, \frac{x}{\varepsilon}\right) d x=\int_{\Omega \times Y} f(x, y) g(x, y) d x d y .
$$

4. Let $f$ be an admissible function and $g \in \mathcal{C}(\Omega \times Y)$. Show that $f g$ is admissible.

Exercise 2.2. Let $\left(u_{\varepsilon}\right)_{\varepsilon}$ be a sequence of functions in $L^{2}(\Omega)$ that $L^{2}$ strongly converges to $u_{0} \in L^{2}(\Omega)$. Show that

$$
u_{\varepsilon} \rightarrow u_{0} \text { two scale. }
$$

Exercise 2.3. Let $u_{0}(x, y)$ be an admissible function.

1. Show that $u_{\varepsilon}(x)=u_{0}\left(x, \frac{x}{\varepsilon}\right)$ two-scale converges to $u_{0}$.

2. Let $v_{\varepsilon}=u_{0}\left(x, \frac{x}{\varepsilon^{2}}\right)$. Show that $\left(v_{\varepsilon}\right)_{\varepsilon}$ two-scale converges to $v_{0}(x)=\int_{Y} u_{0}(x, y) d y$. 
3. More generaly, show that any multiscale expansion

$$
u_{\varepsilon}(x)=u_{0}\left(x, \frac{x}{\varepsilon}\right)+\varepsilon u_{1}\left(x, \frac{x}{\varepsilon}\right)+\cdots+\varepsilon^{n} u_{n}\left(x, \frac{x}{\varepsilon}\right)
$$

where the $u_{i}$ are supposed to be admissible, two-scale converges to $u_{0}(x, y)$.

Exercise 2.4. Let $\left(u_{\varepsilon}\right)_{\varepsilon}$ a sequence that two-scale converges to $u_{0}$. Show (using Proposition 2.2) that

$$
\lim _{\varepsilon \rightarrow 0} \int_{\Omega} u_{\varepsilon}(x) \psi\left(x, \frac{x}{\varepsilon}\right) d x=\int_{\Omega} u_{0}(x, y) \psi(x, y) d x d y
$$

for all test functions $\psi$ that are admissible (and not only smooth).

Exercise 2.5. Orthogonal of divergence free functions.

Let

$$
V_{\sharp}=\left\{u \in L^{2}\left(Y, \mathbb{R}^{d}\right) \text { such that } \operatorname{div} u=0 \text { in the sense of } \mathscr{D}^{\prime}(Y)\right\} .
$$

Using the decomposition in Fourier series of a function $u \in V_{\sharp}$, show that

$$
V_{\sharp}^{\perp}=\left\{\psi \in L^{2}(Y) \text { such that } \exists \phi \in H_{\sharp}^{1}(Y), \psi=\nabla \phi\right\} .
$$

Exercise 2.6. Show the following proposition.

Proposition 2.3. Let $\left(u_{\varepsilon}\right)_{\varepsilon>0}$ be a sequence bounded in $L^{2}(\Omega)$ such that $\left(\varepsilon \nabla u_{\varepsilon}\right)_{\varepsilon>0}$ is bounded in $L^{2}(\Omega)$. Show that there exists $u_{0}(x, y) \in L^{2}(\Omega \times Y)$ such that, up to the extraction of a subsequence, one has

$$
\begin{aligned}
& u_{\varepsilon} \rightarrow u_{0} \text { two-scale, } \\
& \varepsilon \nabla u_{\varepsilon} \rightarrow \nabla_{y} u_{0}(x, y) \text { two-scale. }
\end{aligned}
$$

\section{Application to Linear 2nd Order Elliptic Equations}

We now turn to the homogenization of the model problem and generalize the method to classical second order elliptic PDEs. As we shall see, the main strategy consists in the following methodology:

- find a bound in $H^{1}$ for the sequence $\left(u_{\varepsilon}\right)_{\varepsilon}$;

- extract a subsequence and apply Theorem 2.2 ;

- identify the problems solved by $u_{0}$ and $u_{1}$;

- show that the whole sequence converges;

- give sufficient conditions to get the strong convergence and prove the multiscale expansion.

\subsection{Homogenization of 2nd order elliptic problems}

We thus consider again the problem (1.1), namely

$$
\left\{\begin{array}{l}
-\operatorname{div}\left(A\left(\frac{x}{\varepsilon}\right) \nabla u_{\varepsilon}(x)\right)=f(x) \text { in } \Omega, \\
u_{\varepsilon}=0 \text { on } \partial \Omega
\end{array}\right.
$$

where $\Omega$ is bounded and $A$ satisfies the uniform coerciveness assumption (1.2). Let us write the variational formulation associated to this problem. We take a test function $\phi \in H_{0}^{1}(\Omega)$, multiply the equation by $\phi$ and integrate by parts to get

$$
\int_{\Omega}\left(A\left(\frac{x}{\varepsilon}\right) \nabla_{x} u_{\varepsilon}(x), \nabla_{x} \phi(x)\right) d x=\int_{\Omega} f(x) \phi(x) d x .
$$

As we have already pointed out, the sequence $\left(u_{\varepsilon}\right)_{\varepsilon}$ is uniformly bounded and one has the bound (obtained by taking $\phi=u_{\varepsilon}$ in the preceding variational formulation)

$$
\left\|u_{\varepsilon}\right\|_{H^{1}} \lesssim\|f\|_{L^{2}}
$$

Therefore, Theorem 2.2 applies and, up to the extraction of a subsequence (that we still denote by $\left(u_{\varepsilon}\right)_{\varepsilon}$ for simplicity) one can assume that

$$
\begin{aligned}
& u_{\varepsilon} \rightarrow u_{0} \text { weakly in } H^{1}(\Omega), \\
& u_{\varepsilon} \rightarrow u_{0} \text { strongly in } L^{2}(\Omega), \\
& u_{\varepsilon} \rightarrow u_{0} \text { two-scale, } \\
& \nabla u_{\varepsilon} \rightarrow \nabla_{x} u_{0}(x)+\nabla_{y} u_{1}(x, y) \text { two-scale, }
\end{aligned}
$$

where $u_{0} \in H_{0}^{1}(\Omega)$ and $u_{1} \in L^{2}\left(\Omega, H_{\sharp}^{1}(Y) / \mathbb{R}\right)$. 
The idea consists in taking a suitable test function in (3.2) and use the convergences above to pass to the limit. Namely, we consider $\phi_{0} \in \mathscr{D}(\Omega)$ and $\phi_{1} \in \mathscr{D}\left(\Omega, \mathcal{C}_{\sharp}^{\infty}(Y) / \mathbb{R}\right)$ and use the test function

$$
\phi(x)=\phi_{0}(x)+\varepsilon \phi_{1}\left(x, \frac{x}{\varepsilon}\right)
$$

in (3.2).

We obtain, since $\nabla_{x} \phi(x)=\nabla_{x} \phi_{0}(x)+\varepsilon\left(\nabla_{x} \phi_{1}\right)\left(x, \frac{x}{\varepsilon}\right)+\left(\nabla_{y} \phi_{1}\right)\left(x, \frac{x}{\varepsilon}\right)$,

$$
\begin{gathered}
\int_{\Omega}\left(A\left(\frac{x}{\varepsilon}\right) \nabla_{x} u_{\varepsilon}(x), \nabla_{x} \phi_{0}(x)+\varepsilon\left(\nabla_{x} \phi_{1}\right)\left(x, \frac{x}{\varepsilon}\right)+\left(\nabla_{y} \phi_{1}\right)\left(x, \frac{x}{\varepsilon}\right)\right) d x \\
=\int_{\Omega} f(x)\left(\phi_{0}(x)+\varepsilon \phi_{1}\left(x, \frac{x}{\varepsilon}\right)\right) d x .
\end{gathered}
$$

We now pass to the limit in each term. Let us begin with the right-hand side. Since $\phi_{1} \in L^{\infty}(\Omega \times Y)$ we easily have

$$
\lim _{\varepsilon \rightarrow 0} \int_{\Omega} f(x)\left(\phi_{0}(x)+\varepsilon \phi_{1}\left(x, \frac{x}{\varepsilon}\right)\right) d x=\int_{\Omega} f(x) \phi_{0}(x) d x .
$$

Similarly, since $A \in L^{\infty}(Y)$, and $\left(u_{\varepsilon}\right)_{\varepsilon}$ is uniformly bounded in $H^{1}(\Omega)$, we have

$$
\lim _{\varepsilon \rightarrow 0} \int_{\Omega}\left(A\left(\frac{x}{\varepsilon}\right) \nabla_{x} u_{\varepsilon}(x), \varepsilon\left(\nabla_{x} \phi_{1}\right)\left(x, \frac{x}{\varepsilon}\right)\right) d x=0 .
$$

For the first term, we write

$$
\int_{\Omega}\left(A\left(\frac{x}{\varepsilon}\right) \nabla_{x} u_{\varepsilon}(x), \nabla_{x} \phi_{0}(x)\right) d x=\int_{\Omega}\left(\nabla_{x} u_{\varepsilon}(x), A^{t}\left(\frac{x}{\varepsilon}\right) \nabla_{x} \phi_{0}(x)\right) d x,
$$

and, since the function $(x, y) \mapsto A^{t}(y) \nabla_{x} \phi_{0}(x)$ is admissible (see Exercise 2.1 of chapter 2), we obtain

$$
\begin{aligned}
\lim _{\varepsilon \rightarrow 0} & \int_{\Omega}\left(A\left(\frac{x}{\varepsilon}\right) \nabla_{x} u_{\varepsilon}(x), \nabla_{x} \phi_{0}(x)\right) d x \\
& =\int_{\Omega \times Y}\left(\nabla_{x} u_{0}(x)+\nabla_{y} u_{1}(x, y), A^{t}(y) \nabla_{x} \phi_{0}(x)\right) d x d y \\
& =\int_{\Omega \times Y}\left(A(y)\left(\nabla_{x} u_{0}(x)+\nabla_{y} u_{1}(x, y)\right), \nabla_{x} \phi_{0}(x)\right) d x d y .
\end{aligned}
$$

The last term is handled in the same manner. Indeed

$$
\begin{gathered}
\int_{\Omega}\left(A\left(\frac{x}{\varepsilon}\right) \nabla_{x} u_{\varepsilon}(x),\left(\nabla_{y} \phi_{1}\right)\left(x, \frac{x}{\varepsilon}\right)\right) d x \\
=\int_{\Omega}\left(\nabla_{x} u_{\varepsilon}(x), A^{t}\left(\frac{x}{\varepsilon}\right)\left(\nabla_{y} \phi_{1}\right)\left(x, \frac{x}{\varepsilon}\right)\right) d x,
\end{gathered}
$$

and

$$
\begin{aligned}
\lim _{\varepsilon \rightarrow 0} & \int_{\Omega}\left(A\left(\frac{x}{\varepsilon}\right) \nabla_{x} u_{\varepsilon}(x),\left(\nabla_{y} \phi_{1}\right)\left(x, \frac{x}{\varepsilon}\right)\right) d x \\
& =\int_{\Omega \times Y}\left(\nabla_{x} u_{0}(x)+\nabla_{y} u_{1}(x, y), A^{t}(y) \nabla_{y} \phi_{1}(x, y)\right) d x d y \\
& =\int_{\Omega \times Y}\left(A(y)\left(\nabla_{x} u_{0}(x)+\nabla_{y} u_{1}(x, y)\right), \nabla_{y} \phi_{1}(x, y)\right) d x d y
\end{aligned}
$$

where we have made use of the fact that the function $(x, y) \mapsto A^{t}(y) \nabla_{y} \phi_{1}(x, y)$ is admissible (it is, in fact, in $\left.L_{\sharp}^{2}(Y, \mathcal{C}(\Omega))\right)$.

Collecting together the preceding results we obtain the limiting variational formulation

$$
\begin{aligned}
& \int_{\Omega \times Y}\left(A(y)\left(\nabla_{x} u_{0}(x)+\nabla_{y} u_{1}(x, y)\right), \nabla_{x} \phi_{0}(x)+\nabla_{y} \phi_{1}(x, y)\right) d x d y \\
& \quad=\int_{\Omega} f(x) \phi_{0}(x) d x .
\end{aligned}
$$

\subsection{Existence and uniqueness}

The nice thing with the method shown above is that it gives the homogenized variational formulation directly. It is also very natural and knowing Theorem 2.2 makes very clear and intuitive what should be the expected result. 
However, some work still needs to be done, namely, recovering the cell and homogenized problem, after having proved that the preceding formula provides us with a variational formulation that possesses a unique solution.

We first start by using a density argument to symmetrize the problem (between the unknown and the test functions). Namely, by density, we easily see that (3.5) also holds for all $\phi_{0} \in H_{0}^{1}(\Omega)$ and all $\phi_{1} \in L^{2}\left(\Omega, H_{\sharp}^{1}(Y) / \mathbb{R}\right)$. We now turn to the existence and uniqueness of the solution to the variational formulation

$$
\begin{aligned}
& \text { Find }\left(u_{0}, u_{1}\right) \in H_{0}^{1}(\Omega) \times L^{2}\left(\Omega, H_{\sharp}^{1}(Y) / \mathbb{R}\right) \text { such that } \forall\left(\phi_{0}, \phi_{1}\right) \in H_{0}^{1}(\Omega) \times L^{2}\left(\Omega, H_{\sharp}^{1}(Y) / \mathbb{R}\right) \\
& \int_{\Omega \times Y}\left(A(y)\left(\nabla_{x} u_{0}(x)+\nabla_{y} u_{1}(x, y)\right), \nabla_{x} \phi_{0}(x)+\nabla_{y} \phi_{1}(x, y)\right)=d x d y \int_{\Omega} f(x) \phi_{0}(x) d x .
\end{aligned}
$$

Existence and uniqueness of the solution to this (homogenized) variational formulation follow now from the LaxMilgram Theorem. Indeed, the linear form

$$
l\left(\left(\phi_{0}, \phi_{1}\right)\right)=\int_{\Omega} f(x) \phi_{0}(x) d x
$$

satisfies

$$
\begin{aligned}
\left|l\left(\left(\phi_{0}, \phi_{1}\right)\right)\right| & \leq\|f\|_{L^{2}}\left\|\phi_{0}\right\|_{L^{2}} \\
& \lesssim\|f\|_{L^{2}}\left\|\nabla \phi_{0}\right\|_{L^{2}} \\
& \lesssim\|f\|_{L^{2}}\left\|\left(\phi_{0}, \phi_{1}\right)\right\|_{H_{0}^{1}(\Omega) \times L^{2}\left(\Omega, H_{\sharp}^{1}(Y) / \mathbb{R}\right)} .
\end{aligned}
$$

Next, the bilinear form

$$
a\left(\left(u_{0}, u_{1}\right),\left(v_{0}, v_{1}\right)\right)=\int_{\Omega \times Y}\left(A(y)\left(\nabla_{x} u_{0}+\nabla_{y} u_{1}\right), \nabla_{x} v_{0}+\nabla_{y} v_{1}\right) d x d y
$$

satisfies

$$
\begin{aligned}
& \left|a\left(\left(u_{0}, u_{1}\right),\left(v_{0}, v_{1}\right)\right)\right| \\
& \quad \leq\|A\|_{L^{\infty}}\left(\left\|\nabla_{x} u_{0}\right\|_{L^{2}}+\left\|\nabla_{y} u_{1}\right\|_{L^{2}(\Omega \times Y)}\right)\left(\left\|\nabla_{x} v_{0}\right\|_{L^{2}}+\left\|\nabla_{y} v_{1}\right\|_{L^{2}(\Omega \times Y)}\right) \\
& \quad \leq\|A\|_{L^{\infty}}\left\|\left(u_{0}, u_{1}\right)\right\|_{H_{0}^{1}(\Omega) \times L^{2}\left(\Omega, H_{\sharp}^{1}(Y) / \mathbb{R}\right)}\left\|\left(v_{0}, v_{1}\right)\right\|_{H_{0}^{1}(\Omega) \times L^{2}\left(\Omega, H_{\sharp}^{1}(Y) / \mathbb{R}\right)}
\end{aligned}
$$

and is therefore continuous in $\left(H_{0}^{1}(\Omega) \times L^{2}\left(\Omega, H_{\sharp}^{1}(Y) / \mathbb{R}\right)\right)^{2}$. It is coercive due to the coerciveness assumption $(1.2)$ made on $A$ since

$$
\begin{aligned}
& a\left(\left(u_{0}, u_{1}\right),\left(u_{0}, u_{1}\right)\right) \\
& \quad=\int_{\Omega \times Y}\left(A(y)\left(\nabla_{x} u_{0}(x)+\nabla_{y} u_{1}(x, y)\right), \nabla_{x} u_{0}(x)+\nabla_{y} u_{1}(x, y)\right) d x d y \\
& \quad \geq c\left\|\nabla_{x} u_{0}+\nabla_{y} u_{1}\right\|_{L^{2}(\Omega \times Y)}^{2} .
\end{aligned}
$$

But

$$
\begin{aligned}
\left\|\nabla_{x} u_{0}+\nabla_{y} u_{1}\right\|_{L^{2}(\Omega \times Y)}^{2}= & \int_{\Omega \times Y}\left\|\nabla_{x} u_{0}(x)+\nabla_{y} u_{1}(x, y)\right\|^{2} d x d y \\
= & \int_{\Omega}\left\|\nabla_{x} u_{0}(x)\right\|^{2} d x+\int_{\Omega \times Y}\left\|\nabla_{y} u_{1}(x, y)\right\|^{2} d x d y \\
& +2 \int_{\Omega \times Y} \nabla_{x} u_{0}(x) \cdot \nabla_{y} u_{1}(x, y) d x d y \\
= & \left\|\left(u_{0}, u_{1}\right)\right\|_{H_{0}^{1}(\Omega) \times L^{2}\left(\Omega, H_{\sharp}^{1}(Y) / \mathbb{R}\right)}^{2}
\end{aligned}
$$

since

$$
2 \int_{\Omega \times Y} \nabla_{x} u_{0}(x) \cdot \nabla_{y} u_{1}(x, y) d x d y=2 \int_{\Omega} \nabla_{x} u_{0}(x) \cdot\left(\int_{Y} \nabla_{y} u_{1}(x, y) d y\right) d x=0 .
$$

Eventually, we conclude, since $\left(u_{0}, u_{1}\right)$ are characterized by the homogenized variational formulation above, that the whole sequence $\left(u_{\varepsilon}\right)_{\varepsilon}$ satisfies the convergences of Theorem 2.2, and not only a subsequence.

\subsection{The cell and the homogenized problems}

It remains to find the solution to the cell and homogenized problem that were stated in the Chapter 1 of these notes. To this aim, we simply consider the two problems obtained by taking $\phi_{0}=0$ or $\phi_{1}=0$ respectively.

- $\phi_{0}=0$.

The variational formulation leads in this case to 


$$
\int_{\Omega \times Y}\left(A(y)\left(\nabla_{x} u_{0}(x)+\nabla_{y} u_{1}(x, y)\right), \nabla_{y} \phi_{1}(x, y)\right) d x d y=0 .
$$

Taking $\phi_{1}$ under the form $\phi_{1}(x, y)=\theta(x) \psi(y)$ leads to

$$
\int_{\Omega \times Y} \theta(x)\left(A(y)\left(\nabla_{x} u_{0}(x)+\nabla_{y} u_{1}(x, y)\right), \nabla_{y} \psi(y)\right) d x d y=0
$$

which is the weak form of the problem

$$
\left\{\begin{array}{l}
-\operatorname{div}_{y}\left(A(y)\left(\nabla_{x} u_{0}(x)+\nabla_{y} u_{1}(x, y)\right)\right)=0, \\
u_{1}(x, y) \text { is } Y \text {-periodic. }
\end{array}\right.
$$

This is nothing but the cell problem (1.9).

- $\phi_{1}=0$.

We have now

$$
\int_{\Omega \times Y}\left(A(y)\left(\nabla_{x} u_{0}(x)+\nabla_{y} u_{1}(x, y)\right), \nabla_{x} \phi_{0}(x)\right) d x d y=\int_{\Omega} f(x) \phi_{0}(x) d x
$$

which is the weak form of the problem

$$
\left\{\begin{array}{l}
-\operatorname{div}_{x}\left(\int_{Y}\left(A(y)\left(\nabla_{x} u_{0}(x)+\nabla_{y} u_{1}(x, y)\right)\right) d y\right)=f \text { in } \Omega, \\
u_{0}=0 \text { on } \partial \Omega .
\end{array}\right.
$$

And we recognize the homogenized problem.

Solving $u_{1}$ in terms of $u_{0}$ through the correctors $\omega_{i}$ is eventually done as before.

\subsection{Exercises}

Exercise 3.1. Assume that $u_{1}$ is smooth. Show that

$$
u_{\varepsilon}-u_{0}(x)-\varepsilon u_{1}\left(x, \frac{x}{\varepsilon}\right)
$$

strongly converges to 0 in $H^{1}(\Omega)$.

Hint: Show that

$$
\int_{\Omega}\left(A\left(\frac{x}{\varepsilon}\right)\left(\nabla_{x} u_{\varepsilon}-\nabla_{x} u_{0}-\nabla_{y} u_{1}\right), \nabla_{x} u_{\varepsilon}-\nabla_{x} u_{0}-\nabla_{y} u_{1}\right) d x
$$

tends to 0 as $\varepsilon \rightarrow 0$ by expanding the expression and pass to the limit in each term. Conclude.

Exercise 3.2. Extend all the preceding results to the case where $A:=A(x, y)$ is an admissible function which satisfies the bounds (1.2).

Exercise 3.3. Linear elasticity. We now consider the model of linear elasticity

$$
-\operatorname{div}\left(\sigma_{\varepsilon}\left(u_{\varepsilon}\right)\right)=f \text { in } \Omega
$$

which, for simplicity is supplemented with the homogeneous Dirichlet boundary conditions $u_{\varepsilon}=0$ on $\partial \Omega$. Here $u_{\varepsilon}: \Omega \rightarrow \mathbb{R}^{d}$ is the deformation vector and the Cauchy stress tensor is given by

$$
\sigma_{\varepsilon}(u)=\mu\left(\frac{x}{\varepsilon}\right)\left(\nabla u+\nabla^{t} u\right)+\lambda\left(\frac{x}{\varepsilon}\right) \operatorname{div}(u) \operatorname{Id} .
$$

We also assume that both $\lambda$ and $\mu$ satisfy (1.2). Make the homogenization process as $\varepsilon$ tends to 0 . Express in particular the cell problem and the homogenized equation.

\section{Convergence of the Energy}

\section{$4.1 \Gamma$-convergence}

This chapter is meant to give another point of view on the problem in the important case where $A$ is assumed furthermore to be symmetric. Indeed, the problem that we have worked on up to now can also be written as a minimization problem, using the Dirichlet principle

$$
\left(\mathcal{P}_{\varepsilon}\right) \min _{u \in H_{0}^{1}(\Omega)} \frac{1}{2} \int_{\Omega} A\left(\frac{x}{\varepsilon}\right) \nabla_{x} u(x) \cdot \nabla_{x} u(x) d x-\int_{\Omega} f(x) u(x) d x,
$$

for which $u_{\varepsilon}$ is the solution. It would be convenient to have a notion of convergence of minimization problems that 
would be compatible with the convergence of minimizers (here, the two-scale convergence). This is indeed the framework given by De Giorgi $\Gamma$-convergence. The theory is certainly much more involved than what we present here for the need of these lecture notes. We refer the reader to $[4,8,9]$ for more information about the topic and its application to homogenization problems, as well as the introductory text [5].

Definition 4.1. Let $(X, d)$ be a metric space. Consider for $\varepsilon>0$ a family of functionals

$$
g_{\varepsilon}: X \rightarrow \mathbb{R}
$$

a limiting functional

$$
g_{0}: X \rightarrow \mathbb{R}
$$

and the corresponding minimization problems

$$
\left(\mathcal{P}_{\varepsilon}\right) \min _{u \in X} \mathscr{g}_{\varepsilon}(u), \quad\left(\mathcal{P}_{0}\right) \min _{u \in X} \mathscr{g}_{0}(u) .
$$

We say that the family of problems $\left(\mathcal{P}_{\varepsilon}\right) \Gamma(d)$-converges to $\mathcal{P}_{0}$ if for any $u_{0} \in X$ one has

- $\Gamma$ - lim inf. For every sequence $\left(u_{\varepsilon}\right)_{\varepsilon}$ that converges to $u_{0}$ (for the metric $d$ ), one has

$$
\liminf _{\varepsilon \rightarrow 0} g_{\varepsilon}\left(u_{\varepsilon}\right) \geq g_{0}\left(u_{0}\right)
$$

- $\Gamma-\lim \sup$. There exists a sequence $\left(u_{\varepsilon}\right)_{\varepsilon}$ that converges to $u_{0}$ (for the metric $d$ ), such that

$$
\limsup _{\varepsilon \rightarrow 0} g_{\varepsilon}\left(u_{\varepsilon}\right) \leq g_{0}\left(u_{0}\right) \text {. }
$$

The main motivation for the introduction of this definition is the following theorem.

Theorem 4.1. Let $(X, d)$ be a metric space and a sequence of minimization problems $\left(\mathcal{P}_{\varepsilon}\right)_{\varepsilon>0}$ that $\Gamma(d)$-converges to the minimization problem $\mathcal{P}_{0}$ as in (4.2). Let $\left(u_{\varepsilon}\right)_{\varepsilon}$ a sequence of solutions of the problems $\left(\mathcal{P}_{\varepsilon}\right)$ that converges to $u_{0}$ for the metric $d$. Then $u_{0}$ is a solution of the minimization problem $\mathcal{P}_{0}$ and

$$
\lim _{\varepsilon \rightarrow 0} g_{\varepsilon}\left(u_{\varepsilon}\right)=g_{0}\left(u_{0}\right)
$$

Proof. Since $\left(u_{\varepsilon}\right)_{\varepsilon}$ converges to $u_{0}$ for the metric $d$, the first assertion of $\Gamma$-convergence entails

$$
g_{0}\left(u_{0}\right) \leq \liminf _{\varepsilon \rightarrow 0} g_{\varepsilon}\left(u_{\varepsilon}\right) .
$$

Now let $v_{0} \in X$, and a sequence $\left(v_{\varepsilon}\right)_{\varepsilon}$ which converges to $v_{0}$ such that

$$
\mathscr{g}_{0}\left(v_{0}\right) \geq \limsup _{\varepsilon \rightarrow 0} g_{\varepsilon}\left(v_{\varepsilon}\right)
$$

Such a sequence exists due to the second assertion of $\Gamma$-convergence. Since $u_{\varepsilon}$ is a minimizer of $g_{\varepsilon}$, one has

$$
\mathscr{g}_{\varepsilon}\left(u_{\varepsilon}\right) \leq \mathcal{g}_{\varepsilon}\left(v_{\varepsilon}\right) \text {. }
$$

We therefore deduce

$$
\begin{aligned}
\mathscr{g}_{0}\left(u_{0}\right) & \leq \liminf _{\varepsilon \rightarrow 0} \mathcal{g}_{\varepsilon}\left(u_{\varepsilon}\right) \\
& \leq \limsup _{\varepsilon \rightarrow 0} \mathcal{g}_{\varepsilon}\left(u_{\varepsilon}\right) \\
& \leq \limsup _{\varepsilon \rightarrow 0} \mathcal{g}_{\varepsilon}\left(v_{\varepsilon}\right) \\
& \leq \mathscr{g}_{0}\left(v_{0}\right)
\end{aligned}
$$

which shows that $u_{0}$ is a solution to $\mathcal{P}_{0}$. Taking $v_{0}=u_{0}$ in the preceding inequalities shows the last assertion of the theorem.

Remark 4.1. The $\Gamma$-convergence is a framework which is very well suited for the convergence of minimization problems. Quite remarkably, the definition itself does not use any minimization property of the sequence $\left(u_{\varepsilon}\right)_{\varepsilon}$ or the limit $u_{0}$. Minimization and convergence of minimizers are thus obtained as consequences of the general properties given in the definition.

In the definition of $\Gamma$-convergence, (4.4) may be replaced by an equivalent or weaker statement for which Theorem 4.1 still holds true. We refer the interested reader to [5] for a detailed list of possible statements, but we will make use of the following one in the sequel

For all $\eta>0$, there exists a sequence $\left(u_{\varepsilon}\right)_{\varepsilon}$ that converges to $u_{0}$ (for the metric $d$ ), such that

$$
\limsup _{\varepsilon \rightarrow 0} g_{\varepsilon}\left(u_{\varepsilon}\right) \leq \mathscr{g}_{0}\left(u_{0}\right)+\eta \text {. }
$$


That under (4.3) and (4.5), Theorem 4.1 still holds true is left as an exercise (see Exercise 4.1).

\subsection{Application to homogenization}

From what we have already seen, we set, for $u \in X=H_{0}^{1}(\Omega)$

$$
\mathcal{g}_{\varepsilon}(u)=\frac{1}{2} \int_{\Omega} A\left(\frac{x}{\varepsilon}\right) \nabla_{x} u(x) \cdot \nabla_{x} u(x) d x-\int_{\Omega} f(x) u(x) d x
$$

while the limiting problem involves the homogenized tensor $A_{\text {eff }}$ defined by (1.14)

$$
\mathscr{g}_{0}(u)=\frac{1}{2} \int_{\Omega} A_{\mathrm{eff}} \nabla_{x} u(x) \cdot \nabla_{x} u(x) d x-\int_{\Omega} f(x) u(x) d x .
$$

It remains to choose a metric on $X$. Clearly, the convergence that we proved in the preceding sections is only weak in $H^{1}(\Omega)$ which is not metrizable. We therefore endow $X$ with the (strong) $L^{2}$ distance (see Remark 4.3 below).

The main goal of this Section is to show the following Theorem.

Theorem 4.2. Let $g_{\varepsilon}$ (resp. $g_{0}$ ) be defined by (4.6) (resp. (4.7)), and consider the associated minimization problems $\mathcal{P}_{\varepsilon}$ and $\mathcal{P}_{0}$ given by (4.2). The sequence of minimization problems $\left(\mathcal{P}_{\varepsilon}\right)_{\varepsilon} \Gamma\left(L^{2}\right)$-converges to $\mathcal{P}_{0}$ in $H_{0}^{1}(\Omega)$.

Proof. The proof consists in proving both properties of $\Gamma$-convergence. As we shall see, this sheds a new light on the problem. We therefore decompose the proof into two steps.

$\Gamma-\lim$ inf.

Let $\left(u_{\varepsilon}\right)_{\varepsilon}$ be a sequence in $H_{0}^{1}(\Omega)$ that converges to $u_{0} \in H_{0}^{1}(\Omega)$ for the $L^{2}$ topology. Let $A=\liminf \sin _{\varepsilon \rightarrow 0} \mathscr{g}_{\varepsilon}\left(u_{\varepsilon}\right)$. If $A=+\infty$, there is nothing to prove. Otherwise up to the extraction of a subsequence, we may assume furthermore that

$$
\lim _{\varepsilon \rightarrow 0} g_{\varepsilon}\left(u_{\varepsilon}\right)=A \text {. }
$$

In view of (1.2) we deduce that the sequence $\left(u_{\varepsilon}\right)_{\varepsilon}$ is bounded in $H^{1}$, and applying Theorem 2.2 , there exists $u_{1} \in$ $L^{2}\left(\Omega, H_{\sharp}^{1}\right)$ such that

$$
\nabla u_{\varepsilon} \rightarrow \nabla_{x} u_{0}(x)+\nabla_{y} u_{1}(x, y) \text { two-scale. }
$$

We take now $\psi(x, y) \in \mathscr{D}\left(\Omega, \mathcal{C}_{\sharp}^{\infty}\right)$ an admissible function and expand the non-negative expression

$$
\begin{aligned}
\int_{\Omega} A\left(\frac{x}{\varepsilon}\right)\left(\nabla_{x} u_{\varepsilon}-\nabla_{x} u_{0}-\nabla_{y} \psi\left(x, \frac{x}{\varepsilon}\right)\right) \cdot\left(\nabla_{x} u_{\varepsilon}-\nabla_{x} u_{0}-\nabla_{y} \psi\left(x, \frac{x}{\varepsilon}\right)\right) d x \\
=\int_{\Omega} A\left(\frac{x}{\varepsilon}\right) \nabla_{x} u_{\varepsilon} \cdot \nabla_{x} u_{\varepsilon} \\
\quad-2 \int_{\Omega} A\left(\frac{x}{\varepsilon}\right) \nabla_{x} u_{\varepsilon} \cdot\left(\nabla_{x} u_{0}+\nabla_{y} \psi\left(x, \frac{x}{\varepsilon}\right)\right) d x \\
\quad+\int_{\Omega} A\left(\frac{x}{\varepsilon}\right)\left(\nabla_{x} u_{0}+\nabla_{y} \psi\left(x, \frac{x}{\varepsilon}\right)\right) \cdot\left(\nabla_{x} u_{0}+\nabla_{y} \psi\left(x, \frac{x}{\varepsilon}\right)\right) d x .
\end{aligned}
$$

Due to the preceding results and hypotheses, we infer

$$
\begin{gathered}
\lim _{\varepsilon \rightarrow 0} \int_{\Omega} A\left(\frac{x}{\varepsilon}\right)\left(\nabla_{x} u_{0}+\nabla_{y} \psi\left(x, \frac{x}{\varepsilon}\right)\right) \cdot\left(\nabla_{x} u_{0}+\nabla_{y} \psi\left(x, \frac{x}{\varepsilon}\right)\right) d x \\
=\int_{\Omega \times Y} A(y)\left(\nabla_{x} u_{0}(x)+\nabla_{y} \psi(x, y)\right) \cdot\left(\nabla_{x} u_{0}(x)+\nabla_{y} \psi(x, y)\right) d x d y
\end{gathered}
$$

and

$$
\begin{aligned}
\lim _{\varepsilon \rightarrow 0} \int_{\Omega} A\left(\frac{x}{\varepsilon}\right) \nabla_{x} u_{\varepsilon} \cdot\left(\nabla_{x} u_{0}+\nabla_{y} \psi\left(x, \frac{x}{\varepsilon}\right)\right) d x \\
=\lim _{\varepsilon \rightarrow 0} \int_{\Omega} \nabla_{x} u_{\varepsilon} \cdot A^{t}\left(\frac{x}{\varepsilon}\right)\left(\nabla_{x} u_{0}+\nabla_{y} \psi\left(x, \frac{x}{\varepsilon}\right)\right) d x \\
=\int_{\Omega \times Y}\left(\nabla_{x} u_{0}+\nabla_{y} u_{1}(x, y)\right) \cdot A^{t}(y)\left(\nabla_{x} u_{0}(x)+\nabla_{y} \psi(x, y)\right) d x d y \\
=\int_{\Omega \times Y} A(y)\left(\nabla_{x} u_{0}+\nabla_{y} u_{1}(x, y)\right) \cdot\left(\nabla_{x} u_{0}(x)+\nabla_{y} \psi(x, y)\right) d x d y .
\end{aligned}
$$

Since moreover

$$
\lim _{\varepsilon \rightarrow 0} \int_{\Omega} f(x) u_{\varepsilon}(x) d x=\int_{\Omega} f(x) u_{0}(x) d x,
$$

we therefore deduce 


$$
\begin{aligned}
\liminf _{\varepsilon \rightarrow 0} & g_{\varepsilon}\left(u_{\varepsilon}\right) \\
\geq & \int_{\Omega \times Y} A(y)\left(\nabla_{x} u_{0}(x)+\nabla_{y} u_{1}(x, y)\right) \cdot\left(\nabla_{x} u_{0}(x)+\nabla_{y} \psi(x, y)\right) d x d y \\
& -\frac{1}{2} \int_{\Omega \times Y} A(y)\left(\nabla_{x} u_{0}(x)+\nabla_{y} \psi(x, y)\right) \cdot\left(\nabla_{x} u_{0}(x)+\nabla_{y} \psi(x, y)\right) d x d y \\
& -\int_{\Omega} f(x) u_{0}(x) d x .
\end{aligned}
$$

Taking for $\psi$ a sequence of functions that converges to $u_{1}$ in $L^{2}\left(\Omega, H_{\sharp}^{1}(Y)\right)$, we obtain

$$
\begin{aligned}
\liminf _{\varepsilon \rightarrow 0} g_{\varepsilon}\left(u_{\varepsilon}\right) \geq & \frac{1}{2} \int_{\Omega \times Y} A(y)\left(\nabla_{x} u_{0}+\nabla_{y} u_{1}\right) \cdot\left(\nabla_{x} u_{0}+\nabla_{y} u_{1}\right) d x d y \\
& -\int_{\Omega} f(x) u_{0}(x) d x .
\end{aligned}
$$

The last part of the proof consists in remarking that the right-hand side may be bounded from below since

$$
\begin{aligned}
& \frac{1}{2} \int_{\Omega \times Y} A(y)\left(\nabla_{x} u_{0}+\nabla_{y} u_{1}\right) \cdot\left(\nabla_{x} u_{0}+\nabla_{y} u_{1}\right) d x d y \\
& \quad \geq \min _{u_{1} \in L^{2}\left(\Omega, H_{\sharp}^{1}\right)} \frac{1}{2} \int_{\Omega \times Y} A(y)\left(\nabla_{x} u_{0}+\nabla_{y} u_{1}\right) \cdot\left(\nabla_{x} u_{0}+\nabla_{y} u_{1}\right) d x d y \\
& \quad=\frac{1}{2} \int_{\Omega} A_{\mathrm{eff}} \nabla_{x} u_{0} \cdot \nabla_{x} u_{0} d x .
\end{aligned}
$$

We thus obtain

$$
\liminf _{\varepsilon \rightarrow 0} g_{\varepsilon}\left(u_{\varepsilon}\right) \geq \frac{1}{2} \int_{\Omega} A_{\mathrm{eff}} \nabla_{x} u_{0} \cdot \nabla_{x} u_{0} d x-\int_{\Omega} f(x) u_{0}(x) d x=g_{0}\left(u_{0}\right)
$$

$\Gamma-\lim \sup$.

The proof for this part of $\Gamma$-convergence is sometimes called the construction of a recovery sequence. Indeed, it consists for a given $u_{0} \in H_{0}^{1}(\Omega)$, in finding a suitable sequence $\left(u_{\varepsilon}\right)_{\varepsilon}$ in $H_{0}^{1}(\Omega)$ that converges to $u_{0}$ in $L^{2}$ and such that

$$
\limsup _{\varepsilon \rightarrow 0} g_{\varepsilon}\left(u_{\varepsilon}\right) \leq g_{0}\left(u_{0}\right)
$$

In view of the preceding results, we build from the corrector Eq. (1.9) the microscopic structure $u_{1}$, and a natural recovery sequence would be

$$
u_{\varepsilon}=u_{0}(x)+\varepsilon u_{1}\left(x, \frac{x}{\varepsilon}\right)
$$

However, since $u_{1}$ may not be an admissible function, we need to complexify a little bit the argument. We take $\psi_{1}$ an admissible function in $\mathscr{D}\left(\Omega, \mathcal{C}_{\sharp}^{\infty}(Y)\right)$ and consider

$$
u_{\varepsilon}=u_{0}(x)+\varepsilon \psi_{1}\left(x, \frac{x}{\varepsilon}\right) .
$$

It is easy to check that

$$
\lim _{\varepsilon \rightarrow 0}\left\|u_{\varepsilon}-u_{0}\right\|_{L^{2}}=0 \text {. }
$$

Moreover, since $\nabla_{x} u_{\varepsilon}=\nabla_{x} u_{0}(x)+\left(\nabla_{y} \psi_{1}\right)\left(x, \frac{x}{\varepsilon}\right)+\varepsilon\left(\nabla_{x} \psi_{1}\right)\left(x, \frac{x}{\varepsilon}\right)$ we get by using the two-scale convergence results that

$$
\begin{aligned}
\lim _{\varepsilon \rightarrow 0} \mathcal{g}_{\varepsilon}\left(u_{\varepsilon}\right)= & \frac{1}{2} \int_{\Omega \times Y} A(y)\left(\nabla_{x} u_{0}+\nabla_{y} \psi_{1}\right) \cdot\left(\nabla_{x} u_{0}+\nabla_{y} \psi_{1}\right) d x d y \\
& -\int_{\Omega} f(x) u_{0}(x) d x .
\end{aligned}
$$

Taking now $\psi_{1}$ that converges to $u_{1}$ in $L^{2}\left(\Omega, H_{\sharp}^{1}(Y)\right)$ and remarking that

leads to

$$
g_{0}\left(u_{0}\right)=\frac{1}{2} \int_{\Omega \times Y} A(y)\left(\nabla_{x} u_{0}+\nabla_{y} u_{1}\right) \cdot\left(\nabla_{x} u_{0}+\nabla_{y} u_{1}\right) d x d y-\int_{\Omega} f(x) u_{0}(x) d x,
$$

$$
\forall \eta>0, \exists\left(u_{\varepsilon}\right)_{\varepsilon} \text { in } H_{0}^{1}(\Omega) \text { s.t. } \lim _{\varepsilon \rightarrow 0} g_{\varepsilon}\left(u_{\varepsilon}\right) \leq g_{0}\left(u_{0}\right)+\eta
$$


which is exactly (4.5) and therefore enough to get the $\Gamma$-convergence property.

Remark 4.2. The proof made above used very clearly the fact that the cell problem (1.9) can be also seen as a minimization problem. Namely for a given macroscopic $u_{0}$, the microscopic structure, represented by $u_{1}$ is the one that minimizes

$$
\int_{\Omega \times Y} A(y)\left(\nabla_{x} u_{0}+\nabla_{y} u_{1}\right) \cdot\left(\nabla_{x} u_{0}+\nabla_{y} u_{1}\right) d x d y .
$$

It is indeed easily seen that (1.9) is the variational formulation associated to this minimization problem.

Remark 4.3. As we have already pointed out, the convergence only holds in the strong $L^{2}$ or weak $H^{1}$ senses. Although the weak $H^{1}$ topology is not metrizable, we remark that the definition of $\Gamma$-convergence does not need the topology to be metrizable, but only a notion of convergence of sequences. We therefore could (and some authors do) use the weak $H^{1}$ topology instead in order to prove all the statements before.

\subsection{Exercises}

Exercise 4.1. Show that under assumptions (4.3) and (4.5), Theorem 4.1 still holds true.

Exercise 4.2. Show that in the case where $A$ is symmetric, the solution $u_{1}(x, \cdot)$ to the cell problem (1.9) is indeed the solution to the minimization problem

$$
\min _{\psi \in H_{\sharp}^{1}(Y)} \int_{Y} A(y)\left(\nabla_{x} u_{0}(x)+\nabla_{y} \psi(y)\right) \cdot\left(\nabla_{x} u_{0}(x)+\nabla_{y} \psi(y)\right) d y .
$$

Deduce that $\left(u_{0}, u_{1}\right)$ solve the minimization problem

$$
\min _{\left(\phi_{0}, \phi_{1}\right) \in H_{0}^{1}(\Omega) \times L^{2}\left(\Omega, H_{\sharp}^{1}(Y)\right)} \mathcal{E}\left(\left(\phi_{0}, \phi_{1}\right)\right)
$$

where

$$
\begin{aligned}
\mathscr{E}\left(\left(\phi_{0}, \phi_{1}\right)\right)= & \frac{1}{2} \int_{\Omega \times Y} A(y)\left(\nabla_{x} \phi_{0}(x)+\nabla_{y} \phi_{1}(x, y)\right) \cdot\left(\nabla_{x} \phi_{0}(x)+\nabla_{y} \phi_{1}(x, y)\right) d x d y \\
& -\int_{\Omega} f(x) \phi_{0}(x) d x .
\end{aligned}
$$

Exercise 4.3. Let $A_{\text {eff }}$ be the effective diffusion tensor given by (1.14) in the case where $A$ is symmetric. Show that $\forall \xi \in \mathbb{R}^{d}$

$$
\left(\int_{Y} A^{-1}(y) d y\right)^{-1} \xi \cdot \xi \leq A_{\mathrm{eff}} \xi \cdot \xi \leq\left(\int_{Y} A(y) d y\right) \xi \cdot \xi .
$$

Hint: Use the minimization properties of the cell problem.

Exercise 4.4. As usual, we consider $\Omega$ an open bounded regular set of $\mathbb{R}^{d}$ and $f \in L^{2}(\Omega)$. Let

$$
W: \mathbb{R}^{d} \times \mathbb{R}^{d} \rightarrow \mathbb{R}
$$

be an energy density function and assume

$$
\begin{aligned}
& y \mapsto W(y, \lambda) \text { is } Y \text {-periodic; } \\
& \lambda \mapsto W(y, \lambda) \text { is } \mathcal{C}^{1} \text { and strictly convex in } \mathbb{R}^{d^{2}} ; \\
& |\lambda|^{2} \lesssim W(y, \lambda) \lesssim 1+|\lambda|^{2} \text { uniformly in } y ; \\
& \left|\frac{\partial W}{\partial \lambda}(y, \lambda)\right| \lesssim 1+|\lambda| \text { uniformly in } y .
\end{aligned}
$$

We also set for all $u \in H_{0}^{1}(\Omega)$

$$
\ell_{\varepsilon}(u)=\int_{\Omega} W\left(\frac{x}{\varepsilon}, \nabla_{x} u(x)\right) d x-\int_{\Omega} f(x) u(x) d x .
$$

1. Show that under the preceding hypotheses on $W$, the minimization problem

has a unique solution that we call $u_{\varepsilon}$.

$$
\min _{u \in H_{0}^{1}(\Omega)} \ell_{\varepsilon}(u)
$$

2. Show that $\left(u_{\varepsilon}\right)_{\varepsilon}$ is bounded in $H_{0}^{1}(\Omega)$ and deduce that there exists $u_{0} \in H_{0}^{1}(\Omega)$ and $u_{1} \in L^{2}\left(\Omega, H_{\sharp}^{1}(Y)\right)$ such that, up to a subsequence 


$$
\begin{aligned}
& u_{\varepsilon} \rightarrow u_{0} \text { weakly in } H^{1} \\
& u_{\varepsilon} \rightarrow u_{0} \text { strongly in } L^{2} \\
& u_{\varepsilon} \rightarrow u_{0} \text { two-scale; } \\
& \nabla_{x} u_{\varepsilon} \rightarrow \nabla_{x} u_{0}(x)+\nabla_{y} u_{1}(x, y) \text { two-scale. }
\end{aligned}
$$

The last part of this exercise consists in proving that $\ell_{\varepsilon} \Gamma\left(L^{2}\right)$-converges in $H_{0}^{1}(\Omega)$ to

$$
\ell_{0}(u)=\inf _{\psi_{1} \in L^{2}\left(\Omega, H_{\sharp}^{1}(Y)\right)} \int_{\Omega \times Y} W\left(y, \nabla_{x} u+\nabla_{y} \psi(x, y)\right) d x d y-\int_{\Omega} f(x) u(x) d x .
$$

3. Using

$$
W(\cdot, \lambda) \geq W(\cdot, \mu)+\left\langle\frac{\partial W}{\partial \lambda}(\cdot, \mu), \lambda-\mu\right\rangle
$$

prove that if $\left(v_{\varepsilon}\right)_{\varepsilon}$ is a sequence in $H_{0}^{1}(\Omega)$ that converges in $L^{2}$ to $v_{0} \in H_{0}^{1}(\Omega)$

$$
\liminf _{\varepsilon \rightarrow 0} \ell_{\varepsilon}\left(v_{\varepsilon}\right) \geq \int_{\Omega \times Y} W\left(y, \nabla_{x} v_{0}+\nabla_{y} \psi\right) d x d y-\int_{\Omega} f(x) v_{0}(x) d x,
$$

for some $\psi \in L^{2}\left(\Omega, H_{\sharp}^{1}(Y)\right)$. Deduce that

$$
\liminf _{\varepsilon \rightarrow 0} \ell_{\varepsilon}\left(v_{\varepsilon}\right) \geq \ell_{0}\left(v_{0}\right) .
$$

Hint: Use a sequence $\mu$ of smooth test functions that converges to $\nabla_{x} u_{0}(x)+\nabla_{y} u_{1}(x, y)$ in $L^{2}(\Omega \times Y)$.

4. Let $v_{0}$ in $H_{0}^{1}(\Omega)$, show that for all $\eta>0$ there exists $\left(v_{\varepsilon}\right)_{\varepsilon}$ in $H_{0}^{1}(\Omega)$ such that

$$
\begin{aligned}
& v_{\varepsilon} \rightarrow v_{0} \text { in } L^{2} ; \\
& \lim _{\varepsilon \rightarrow 0} \ell_{\varepsilon}\left(v_{\varepsilon}\right) \leq \ell_{0}\left(v_{0}\right)+\eta .
\end{aligned}
$$

5. Conclude.

\section{Perforated Domains - Porous Media}

\subsection{Setting of the problem}

A porous medium is a domain composed of a solid part in which a fluid (e.g. water) is able to flow inside tiny capillaries. Porous media are usually modelled using Darcy's law, which is usually obtained by some averaging of Stokes flow at the microscopic scale. Indeed, the mathematical modelization of such a phenomenon typically involves a fluid structure interaction, the fluid flowing inside the solid matrix. Due to the very small velocities of the fluid, it is very reasonable to consider a low Reynolds number approximation, i.e. that the fluid is modelled by Stokes equations. We also consider that on the solid part of the domain, a no-slip boundary condition is assumed for the fluid. We explain here how the two-scale convergence process is able to recover Darcy's law from a microscopic Stokes equation. The pioneering works, that formally derived Darcy's law from the microscopic Stokes equations may be found in e.g. $[11,17]$ while the first rigorous proof is probably in [18]. Further extensions may be found in [2, 13].

In what follows, we denote by $\Omega_{\varepsilon}$ the fluid part of $\Omega$. We consider that the fluid obeys Stokes equations

$$
\begin{cases}-\varepsilon^{2} \mu \Delta u_{\varepsilon}+\nabla p_{\varepsilon}=f & \text { in } \Omega_{\varepsilon}, \\ \operatorname{div} u_{\varepsilon}=0 & \text { in } \Omega_{\varepsilon}, \\ u_{\varepsilon}=0 & \text { on } \partial \Omega_{\varepsilon} .\end{cases}
$$

In the preceding equations, we have rescaled the viscosity $\mu$ of the fluid by a factor of $\varepsilon^{2}$. As will be seen later on, this simply comes from the fact that otherwise the velocity $u_{\varepsilon}$ tends to 0 . In other words, the velocity of the fluid is of order $\varepsilon^{2}$ and needs to be rescaled in order to observe a non vanishing limit.

Existence and uniqueness of a solution $\left(u_{\varepsilon}, p_{\varepsilon}\right) \in H_{0}^{1}\left(\Omega_{\varepsilon}, \mathbb{R}^{d}\right) \times L^{2}\left(\Omega_{\varepsilon}\right) / \mathbb{R}$ to (5.1) is a well known result as long as $f \in L^{2}\left(\Omega_{\varepsilon}, \mathbb{R}^{d}\right)$ for instance. We can therefore proceed to the homogenization, that is seeking the limits of $u_{\varepsilon}$ and $p_{\varepsilon}$ as $\varepsilon \rightarrow 0$. Quite strangely, here there is no oscillating coefficients like for the diffusion problem before. Instead, here, the domain will be considered as periodic and quickly oscillating. In particular, the domain changes with $\varepsilon$ and is not fixed in the convergence process.

Indeed, as before, we consider a periodic structure in $\Omega$. In that aim, we assume that the unit cell $Y$ can be decomposed as

$$
Y=Y_{s} \cup Y_{f}, Y_{s} \cap Y_{f}=\emptyset,
$$

where $Y_{s}$ corresponds to the solid part in the unit cell and $Y_{f}$ to the fluid part (see Fig. 5.1). 


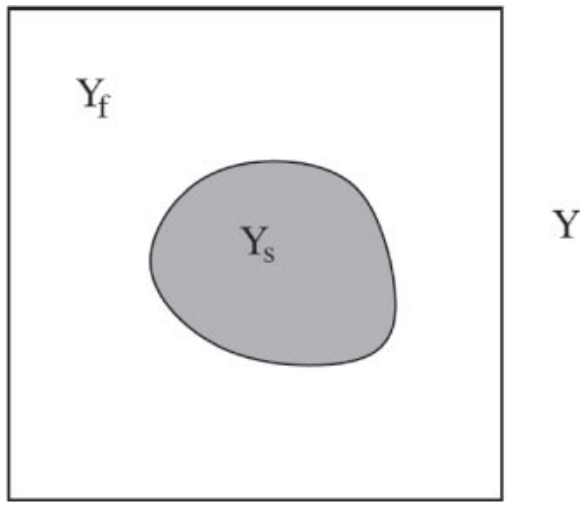

Fig. 5.1. The unit cell of the porous medium is composed of a solid part $Y_{s}$ and a fluid one in which the fluid flows $Y_{f}$.

Scaling down this structure by a factor of $\varepsilon$ and repeating it inside the domain, leads to a periodicly structured domain, as depicted in Fig. 5.2, alternating solid and fluid parts. Namely, viewed from the macroscopic domain, we define the fluid domain as

$$
\Omega_{\varepsilon}=\Omega \backslash \bigcup_{i=1}^{N(\varepsilon)} Y_{s, i}^{\varepsilon}=\Omega \cap \bigcup_{i=1}^{N(\varepsilon)} Y_{f, i}^{\varepsilon}
$$

where each $Y_{f, i}^{\varepsilon}\left(\operatorname{resp} . Y_{s, i}^{\varepsilon}\right)$ is a copy of $\varepsilon Y_{f}$ (resp. $\left.\varepsilon Y_{s}\right)$. The total number of periodic cells $N(\varepsilon)$ satisfies $N(\varepsilon)=$ $|\Omega| \varepsilon^{-d}(1+o(1))$.

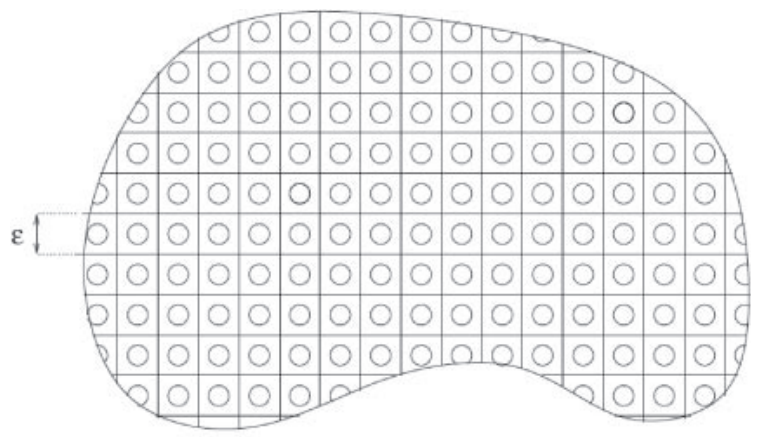

$\Omega$

Fig. 5.2. The domain $\Omega$ tiled by repetitions of $Y$ scaled by a factor $\varepsilon$.
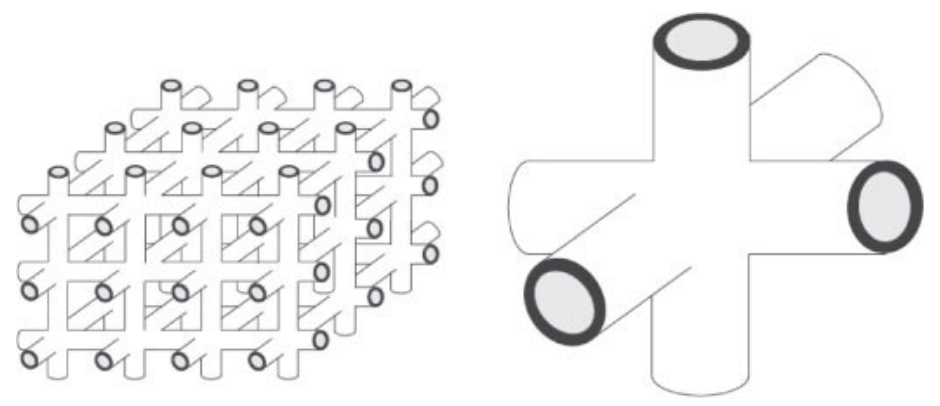

Fig. 5.3. In 3d, it is possible to have a fluid and a solid domains that are both connected. An example is given by the periodic structure shown on the left and made by repetitions of the unit structure to the right (from [15]).

\subsection{Homogenization}

Compared to what we have seen before, we have here an additional difficulty that prevents us from passing to the limit directly on the solution $\left(u_{\varepsilon}, p_{\varepsilon}\right)$. Indeed, the functions are not defined on the same domain since the fluid domain $\Omega_{\varepsilon}$ changes with the value $\varepsilon$. It is, therefore, necessary to extend the solution $u_{\varepsilon}$ and the pressure $p_{\varepsilon}$ suitably before attempting to pass to the limit. 
Since $u_{\varepsilon}=0$ on $\partial \Omega_{\varepsilon}$, we may extend $u_{\varepsilon}$ by 0 inside the solid part, by setting

$$
\tilde{u}_{\varepsilon}(x)=\left\{\begin{array}{l}
u_{\varepsilon}(x) \text { if } x \in \Omega_{\varepsilon}, \\
0 \text { otherwise. }
\end{array}\right.
$$

For $p_{\varepsilon}$ this is slightly more involved. Classically, the pressure is defined up to an additive constant, and we must extend $p_{\varepsilon}$ in such a way that when $p_{\varepsilon}$ is locally constant, it is also constant (with the same constant) inside the solid part. We therefore define for $i \in\{1, \cdots, N(\varepsilon)\}$

$$
\tilde{p}_{\varepsilon}(x)=\left\{\begin{array}{l}
p_{\varepsilon}(x) \text { if } x \in Y_{f, i}^{\varepsilon}, \\
\frac{1}{\left|Y_{f, i}^{\varepsilon}\right|} \int_{Y_{f, i}^{\varepsilon}} p_{\varepsilon} d x \text { in } Y_{s, i}^{\varepsilon} .
\end{array}\right.
$$

\subsection{Convergence Theorem}

This section deals with the two-scale limit of the Stokes equations that we have introduced before. However, the complete proof of the result is rather technical and certainly beyond the scope of these notes. We refer the interested reader to $[2,13,18]$ where it can be found and will give only the key ingredients and arguments. It will be therefore possible to follow the strategy although all of the rigorous details will not be given.

Lemma 5.1. We have the estimates

$$
\begin{aligned}
& \left\|\tilde{u}_{\varepsilon}\right\|_{L^{2}}+\varepsilon\left\|\nabla \tilde{u}_{\varepsilon}\right\|_{L^{2}} \leq C, \\
& \left\|\tilde{p}_{\varepsilon}\right\|_{L^{2} / \mathbb{R}} \leq C .
\end{aligned}
$$

Proof. We first notice that on any $Y_{f, i}^{\varepsilon}$ one has due to Poincaré inequality

$$
\left\|u_{\varepsilon}\right\|_{L^{2}\left(Y_{f, i}^{\varepsilon}\right)} \lesssim \varepsilon\left\|\nabla u_{\varepsilon}\right\|_{L^{2}\left(Y_{f, i}^{\varepsilon}\right)}
$$

from which we deduce

$$
\left\|\tilde{u}_{\varepsilon}\right\|_{L^{2}\left(Y_{i}^{\varepsilon}\right)} \lesssim \varepsilon\left\|\nabla \tilde{u}_{\varepsilon}\right\|_{L^{2}\left(Y_{i}^{\varepsilon}\right)}
$$

which by summing over all cells gives

$$
\left\|\tilde{u}_{\varepsilon}\right\|_{L^{2}(\Omega)} \lesssim \varepsilon\left\|\nabla \tilde{u}_{\varepsilon}\right\|_{L^{2}(\Omega)} .
$$

Now, multiplying the original Stokes equation by $u_{\varepsilon}$ and integrating by parts over $\Omega_{\varepsilon}$, we obtain

$$
\varepsilon^{2} \mu \int_{\Omega_{\varepsilon}}\left|\nabla u_{\varepsilon}\right|^{2} d x-\int_{\Omega_{\varepsilon}} p_{\varepsilon} \operatorname{div} u_{\varepsilon} d x+\int_{\partial \Omega_{\varepsilon}} p_{\varepsilon} u_{\varepsilon} \cdot n d \sigma=\int_{\Omega_{\varepsilon}} f \cdot u_{\varepsilon} d x .
$$

Since $\operatorname{div} u_{\varepsilon}=0$ and $\left.u_{\varepsilon}\right|_{\partial \Omega_{\varepsilon}}=0$, we get, using the extension

$$
\begin{aligned}
\mu\left\|\varepsilon \nabla u_{\varepsilon}\right\|_{L^{2}\left(\Omega_{\varepsilon}\right)}^{2} & =\mu\left\|\varepsilon \nabla \tilde{u}_{\varepsilon}\right\|_{L^{2}(\Omega)}^{2} \\
& \leq\|f\|_{L^{2}}\left\|u_{\varepsilon}\right\|_{L^{2}\left(\Omega_{\varepsilon}\right)} \\
& =\|f\|_{L^{2}}\left\|\tilde{u}_{\varepsilon}\right\|_{L^{2}(\Omega)} \\
& \lesssim \varepsilon\|f\|_{L^{2}}\left\|\nabla \tilde{u}_{\varepsilon}\right\|_{L^{2}(\Omega)}
\end{aligned}
$$

due to (5.5), from which we deduce (5.3).

The estimate for the pressure is trickier. We refer the interested reader to the original proof by Tartar [18] that was further developed in $[2,13]$.

We are now in a position to prove the following Theorem.

Theorem 5.1. The sequence $\left(\tilde{u}_{\varepsilon}, \tilde{p}_{\varepsilon}\right)$ two-scale converges to the unique solution $\left(u_{0}(x, y), p(x)\right)$ of

$$
\begin{cases}-\mu \Delta_{y} u_{0}+\nabla_{y} p_{1}=f-\nabla_{x} p & \text { in } \Omega \times Y_{f}, \\ \operatorname{div}_{y} u_{0}(x, y)=0 & \text { in } \Omega \times Y_{f}, \\ \operatorname{div}_{x}\left(\int_{Y} u_{0}(x, y) d y\right)=0 & \text { in } \Omega, \\ u_{0}(x, y)=0 & \text { in } \Omega \times Y_{s}, \\ \left(\int_{Y} u_{0}(x, y) d y\right) \cdot n=0 & \text { on } \partial \Omega, \\ y \mapsto u_{0}(x, y), p_{1}(x, y) & \text { is } Y \text {-periodic. }\end{cases}
$$

Remark 5.1. The two-scale homogenized Stokes problem is sometimes called the two-pressure Stokes system as it 
involves the macroscopic pressure $p$ and a microscopic pressure $p_{1}$ which takes into account the microscopic structure. Eliminating the $y$ variable will lead to Darcy's law as we shall see below.

Proof of Theorem 5.1.

The bounds obtained in Lemma 5.1 allow us to extract a subsquence from $\left(\tilde{u}_{\varepsilon}\right)_{\varepsilon}$ (that we still denote by $\left.\left(\tilde{u}_{\varepsilon}\right)_{\varepsilon}\right)$ such that

$$
\begin{aligned}
& \tilde{u}_{\varepsilon} \rightarrow u_{0}(x, y) \text { two-scale, } \\
& \varepsilon \nabla_{x} \tilde{u}_{\varepsilon} \rightarrow \nabla_{y} u_{0}(x, y) \text { two-scale. }
\end{aligned}
$$

(Notice that we have used the result of Exercise 2.6). Now, taking a smooth function $\psi(x, y) \in \mathscr{D}\left(\Omega, \mathcal{C}_{\sharp}^{\infty}(Y)\right.$ ) which is supported in $\Omega \times Y_{s}$, we have

$$
0=\int_{\Omega} \tilde{u}_{\varepsilon}(x) \psi\left(x, \frac{x}{\varepsilon}\right) d x \rightarrow \int_{\Omega \times Y} u_{0}(x, y) \psi(x, y) d x d y,
$$

from which we deduce that

$$
u_{0}(x, y)=0 \text { in } \Omega \times Y_{s} .
$$

Moreover since $\operatorname{div} \tilde{u}_{\varepsilon}=0$, we have

$$
\begin{aligned}
0 & =\int_{\Omega} \operatorname{div} \tilde{u}_{\varepsilon} \psi\left(x, \frac{x}{\varepsilon}\right) d x \\
& =-\int_{\Omega} \tilde{u}_{\varepsilon} \cdot\left(\nabla_{x} \psi+\frac{1}{\varepsilon} \nabla_{y} \psi\right)\left(x, \frac{x}{\varepsilon}\right) d x .
\end{aligned}
$$

Multiplying by $\varepsilon$ and passing to the limit $\varepsilon \rightarrow 0$ leads to

$$
0=\int_{\Omega \times Y} u_{0}(x, y) \cdot \nabla_{y} \psi(x, y) d x d y
$$

which means $\operatorname{div}_{y} u_{0}=0$. Taking now a test function $\psi \in H^{1}(\Omega)$ which does not depend on the $y$ variable (thus such that $\operatorname{div}_{y} \psi=0$ ), and does not vanish on the boundary $\partial \Omega$; we also have after integrating by parts and using $\left.\tilde{u}_{\varepsilon}\right|_{\partial \Omega}=0$

$$
0=\int_{\Omega} \tilde{u}_{\varepsilon} \cdot \nabla_{x} \psi\left(x, \frac{x}{\varepsilon}\right) d x
$$

which, passing to the limit leads to

$$
\operatorname{div}_{x}\left(\int_{Y} u_{0}(x, y) d y\right)=0
$$

and

$$
\left(\int_{Y} u_{0}(x, y) d y\right) \cdot n=0 \text { on } \partial \Omega .
$$

As far as the pressure is concerned, the compactness theorem leads to the existence of $p_{0} \in L^{2}(\Omega \times Y) / \mathbb{R}$ such that

$$
\tilde{p}_{\varepsilon} \rightarrow p_{0} \text { two-scale. }
$$

We take the momentum equation and multiply it by $\varepsilon \psi\left(x, \frac{x}{\varepsilon}\right)$ where $\psi(x, y)$ is a smooth vector valued $Y$-periodic function. Integrating by parts, we get

$$
\begin{aligned}
& \varepsilon^{3} \mu \int_{\Omega} \nabla \tilde{u}_{\varepsilon} \cdot \nabla_{x} \psi\left(x, \frac{x}{\varepsilon}\right) d x+\varepsilon^{2} \mu \int_{\Omega} \nabla \tilde{u}_{\varepsilon} \cdot \nabla_{y} \psi\left(x, \frac{x}{\varepsilon}\right) d x \\
& -\varepsilon \int_{\Omega} \tilde{p}_{\varepsilon} \operatorname{div}_{x} \psi\left(x, \frac{x}{\varepsilon}\right) d x-\int_{\Omega} \tilde{p}_{\varepsilon} \operatorname{div}_{y} \psi\left(x, \frac{x}{\varepsilon}\right) d x \\
& =\varepsilon \int_{\Omega} f(x) \cdot \psi\left(x, \frac{x}{\varepsilon}\right) d x .
\end{aligned}
$$

Passing to the limit $\varepsilon \rightarrow 0$ gives

$$
\int_{\Omega \times Y} p_{0}(x, y) \operatorname{div}_{y} \psi(x, y) d x d y=0,
$$

which shows that $p_{0}$ does not depend on the $y$ variable. There exists $p(x) \in L^{2}(\Omega) / \mathbb{R}$ such that

$$
p_{0}(x, y)=p(x) .
$$

Eventually, we need to recover the homogenized problem. The strategy consists in multiplying the momentum equation by a test function which shares the same characteristics as $u_{0}$. Therefore we now take $\psi(x, y)$ a vector valued test function that satisfies 


$$
\begin{aligned}
& \operatorname{div}_{y} \psi(x, y)=0 \text { on } \Omega \times Y, \\
& \operatorname{div}_{x}\left(\int_{Y} \psi(x, y) d y\right)=0, \\
& \left(\int_{Y} \psi(x, y) d y\right) \cdot n=0 \text { on } \partial \Omega, \\
& \psi(x, y)=0 \text { in } \Omega \times Y_{s} .
\end{aligned}
$$

Multiplying the original Stokes equation by $\psi\left(x, \frac{x}{\varepsilon}\right)$ we get after integrating by parts:

$$
\begin{aligned}
& \varepsilon^{2} \mu \int_{\Omega} \nabla \tilde{u}_{\varepsilon} \cdot \nabla_{x} \psi\left(x, \frac{x}{\varepsilon}\right) d x+\varepsilon \mu \int_{\Omega} \nabla \tilde{u}_{\varepsilon} \cdot \nabla_{y} \psi\left(x, \frac{x}{\varepsilon}\right) d x \\
& \quad-\int_{\Omega} \tilde{p}_{\varepsilon} \operatorname{div}_{x} \psi\left(x, \frac{x}{\varepsilon}\right) d x-\frac{1}{\varepsilon} \int_{\Omega} \tilde{p}_{\varepsilon} \operatorname{div}_{y} \psi\left(x, \frac{x}{\varepsilon}\right) d x \\
& =\int_{\Omega} f(x) \cdot \psi\left(x, \frac{x}{\varepsilon}\right) d x .
\end{aligned}
$$

Using the assumptions above, we infer

$$
\begin{gathered}
\varepsilon^{2} \mu \int_{\Omega} \nabla \tilde{u}_{\varepsilon} \cdot \nabla_{x} \psi\left(x, \frac{x}{\varepsilon}\right) d x+\varepsilon \mu \int_{\Omega} \nabla \tilde{u}_{\varepsilon} \cdot \nabla_{y} \psi\left(x, \frac{x}{\varepsilon}\right) d x \\
-\int_{\Omega} \tilde{p}_{\varepsilon} \operatorname{div}_{x} \psi\left(x, \frac{x}{\varepsilon}\right) d x=\int_{\Omega} f(x) \cdot \psi\left(x, \frac{x}{\varepsilon}\right) d x,
\end{gathered}
$$

and we may pass to the (two-scale) limit as $\varepsilon \rightarrow 0$ to get

which further simplifies into

$$
\begin{aligned}
& \mu \int_{\Omega \times Y} \nabla_{y} u_{0} \cdot \nabla_{y} \psi(x, y) d x d y-\int_{\Omega \times Y} p(x) \operatorname{div}_{x} \psi(x, y) d x d y \\
& \quad=\int_{\Omega \times Y} f(x) \cdot \psi(x, y) d x d y,
\end{aligned}
$$

$$
\mu \int_{\Omega \times Y} \nabla_{y} u_{0}(x, y) \cdot \nabla_{y} \psi(x, y) d x d y=\int_{\Omega \times Y} f(x) \cdot \psi(x, y) d x d y .
$$

By density, the preceding variational formulation holds for any test function $\psi$ in the Hilbert space

$$
\begin{array}{r}
V=\left\{\psi(x, y) \in L^{2}\left(\Omega, H_{\sharp}^{1}(Y)\right) \text { s.t. } \psi(x, y)=0 \text { on } \Omega \times Y_{s}, \operatorname{div}_{y} \psi(x, y)=0,\right. \\
\left.\operatorname{div}_{x}\left(\int_{Y} \psi(x, y) d y\right)=0,\left(\int_{Y} \psi(x, y) d y\right) \cdot n=0 \text { on } \partial \Omega\right\} .
\end{array}
$$

The Lax-Milgram Theorem applies to prove that the variational formulation (5.8) has a unique solution in $u_{0} \in V$. This characterizes the limit and therefore the whole sequence $\left(\tilde{u}_{\varepsilon}\right)_{\varepsilon}$ two-scale converges to $u_{0}$.

Eventually, in order to recover the pressure term, we need to characterize the orthogonal (with respect to the $L^{2}(\Omega \times Y)$ scalar product) of $V$. This was done in [2], where it is shown that

$$
V^{\perp^{2}}=\left\{\nabla_{x} q_{0}(x)+\nabla_{y} q_{1}(x, y) \text { where } q_{0} \in L^{2}(\Omega) / \mathbb{R}, q_{1} \in L^{2}\left(\Omega, L_{\sharp}^{2}\left(Y_{f}\right) / \mathbb{R}\right\} .\right.
$$

(Notice that this is a subspace of $L^{2}\left(\Omega, H^{-1}(Y)\right.$ ). ) We obtain as a consequence the existence of $q_{0} \in L^{2}(\Omega) / \mathbb{R}$ and $q_{1} \in L^{2}\left(\Omega, L_{\sharp}^{2}\left(Y_{f}\right) / \mathbb{R}\right.$ that satisfy

$$
\begin{aligned}
& \mu \int_{\Omega \times Y} \nabla_{y} u_{0} \cdot \nabla_{y} \psi(x, y) d x d y-\int_{\Omega \times Y} p(x) \operatorname{div}_{x} \psi(x, y) d x d y \\
& \quad-\int_{\Omega \times Y} q_{1}(x, y) \operatorname{div}_{y} \psi(x, y) d x d y=\int_{\Omega \times Y} f(x) \cdot \psi(x, y) d x d y .
\end{aligned}
$$

This would give the existence of $p\left(=q_{0}\right)$ and $p_{1}\left(=q_{1}\right)$ in Theorem 5.1 except that we need to prove that $q_{0}$ is indeed the two-scale limit of $\left(p_{\varepsilon}\right)_{\varepsilon}$. In order to do so, we take a test function $\psi(x, y)$ which satisfies only $\operatorname{div}_{y} \psi=0$ into the momentum equation. We deduce from the calculation before that

This permits us to deduce that

$$
\begin{aligned}
& \mu \int_{\Omega \times Y} \nabla_{y} u_{0} \cdot \nabla_{y} \psi(x, y) d x d y-\int_{\Omega \times Y} p(x) \operatorname{div}_{x} \psi(x, y) d x d y \\
& \quad=\int_{\Omega \times Y} f(x) \cdot \psi(x, y) d x d y .
\end{aligned}
$$




$$
\int_{\Omega \times Y} q_{0}(x) \operatorname{div}_{x} \psi(x, y) d x d y=\int_{\Omega \times Y} p(x) \operatorname{div}_{x} \psi(x, y) d x d y
$$

or, in other words, that $q_{0}=p$.

\subsection{The cell and the homogenized problem}

It is also possible to proceed exactly in the same spirit as in the preceding chapters of this book, namely to identify the cell and the homogenized problems.

Cell problem: We first remark that if we take $x$ as a fixed parameter, $\left(u_{0}, p_{1}\right)$ satifies a Stokes equation on the unit cell

$$
\left\{\begin{array}{l}
-\mu \Delta_{y} u_{0}+\nabla_{y} p_{1}=f-\nabla_{x} p \text { for } y \in Y_{f} \\
\operatorname{div}_{y} u_{0}=0 \text { for } y \in Y_{f} \\
u_{0}=0 \text { on } \partial Y_{f} \\
y \mapsto u_{0}(x, y) \text { periodic, } \\
y \mapsto p_{1}(x, y) \text { periodic. }
\end{array}\right.
$$

Therefore, introducing the correctors $\left(\omega_{j}(y), q_{j}(y)\right)_{1 \leq j \leq d}$ solutions to

$$
\left\{\begin{array}{l}
-\mu \Delta_{y} \omega_{j}+\nabla_{y} q_{j}=e_{j} \text { for } y \in Y_{f} \\
\operatorname{div}_{y} \omega_{j}=0 \text { for } y \in Y_{f} \\
\omega_{j}=0 \text { on } \partial Y_{f} \\
y \mapsto \omega_{j}(y) \text { periodic, } \\
y \mapsto q_{j}(y) \text { periodic. }
\end{array}\right.
$$

(we leave to the reader the existence and uniqueness of $\left.\left(\omega_{j}, q_{j}\right) \in H_{\sharp}^{1}\left(Y_{f}\right) \times L_{\sharp}^{2}\left(Y_{f}\right)\right)$ we deduce that

$$
\begin{aligned}
& u_{0}(x, y)=\sum_{j=1}^{d}\left(f_{j}(x)-\frac{\partial p}{\partial x_{j}}(x)\right) \omega_{j}(y), \\
& p_{1}(x, y)=\sum_{j=1}^{d}\left(f_{j}(x)-\frac{\partial p}{\partial x_{j}}(x)\right) q_{j}(y) .
\end{aligned}
$$

Homogenized equation: We plug the preceding expression into the last equations of our problem

$$
\begin{cases}\operatorname{div}_{x}\left(\int_{Y} u_{0}(x, y) d y\right)=0 & \text { in } \Omega \\ \left(\int_{Y} u_{0}(x, y) d y\right) \cdot n=0 & \text { on } \partial \Omega\end{cases}
$$

to get the homogenized problem, namely

$$
\left\{\begin{array}{l}
\operatorname{div}_{x}\left(\sum_{j=1}^{d}\left(f_{j}(x)-\frac{\partial p}{\partial x_{j}}(x)\right) \int_{Y} \omega_{j}(y) d y\right)=0 \quad \text { in } \Omega, \\
\left(\sum_{j=1}^{d}\left(f_{j}(x)-\frac{\partial p}{\partial x_{j}}(x)\right) \int_{Y} \omega_{j}(y) d y\right) \cdot n=0 \quad \text { on } \partial \Omega .
\end{array}\right.
$$

Setting $A$ the matrix defined by

$$
A_{i j}=\int_{Y} \omega_{j}(y) \cdot e_{i} d y
$$

and the velocity

$$
u(x)=\frac{1}{\mu} A\left(f-\nabla_{x} p\right)
$$

we get that $u$ solves

$$
\begin{cases}\operatorname{div}_{x} u(x)=0 & \text { in } \Omega, \\ u(x) \cdot n=0 & \text { on } \partial \Omega,\end{cases}
$$

which in view of (5.15) is nothing but the Darcy's equation where the permeability tensor is given by $\frac{1}{\mu} A$. 


\subsection{Exercises}

Exercise 5.1. We consider the Stokes problem in the domain $\Omega_{\varepsilon}$ described in 5.2

$$
\begin{cases}-\mu \Delta u_{\varepsilon}+\nabla p_{\varepsilon}=f & \text { in } \Omega_{\varepsilon}, \\ \operatorname{div} u_{\varepsilon}=0 & \text { in } \Omega_{\varepsilon}, \\ u_{\varepsilon}=0 & \text { on } \partial \Omega_{\varepsilon},\end{cases}
$$

where we have not scaled the viscosity by the factor $\varepsilon^{2}$. Make the multiscale expansion

$$
u_{\varepsilon}=u_{0}\left(x, \frac{x}{\varepsilon}\right)+\varepsilon u_{1}\left(x, \frac{x}{\varepsilon}\right)+\cdots,
$$

and

$$
p_{\varepsilon}=p_{0}\left(x, \frac{x}{\varepsilon}\right)+\varepsilon p_{1}\left(x, \frac{x}{\varepsilon}\right)+\cdots,
$$

and show that $u_{0}=u_{1}=0$. Therefore the first non vanishing term in the equation is of order $\varepsilon^{2}$. This justifies rescaling the viscosity as in (5.1).

Exercise 5.2. Show that the matrix $A$ defined by (5.14) where the $\omega_{i}$ are given by (5.11) satisfies

$$
A_{i j}=\int_{Y} \omega_{i}(y) \cdot \omega_{j}(y) d y
$$

and is therefore symmetric.

\section{Numerical Methods in Homogenization}

The aim of this section is to describe a few numerical methods that can be used to solve problems with highly oscillating coefficients. In order to do so, we consider again the model problem

$$
\left\{\begin{array}{l}
-\operatorname{div}\left(A\left(\frac{x}{\varepsilon}\right) \nabla u_{\varepsilon}\right)=f \text { in } \Omega, \\
u_{\varepsilon}=0 \text { on } \partial \Omega
\end{array}\right.
$$

where the conductivity tensor $A(y)$ is $Y$ periodic and satisfies the uniform coercivity assumption

$$
\exists \alpha, \beta>0 \text { such that } \forall \xi \in \mathbb{R}^{d}, \alpha|\xi|^{2} \leq(A(y) \xi, \xi) \leq \beta|\xi|^{2},
$$

uniformly for all $y \in Y=(0,1)^{d}$ and $f \in L^{2}(\Omega)$. As usual, existence and uniqueness of the solution $u_{\varepsilon}$ to (6.1) is classical.

At first sight, one can use the usual finite difference or finite element method to solve the problem. However, it is clear that the method needs to catch the oscillations of the coefficients $A\left(\frac{x}{\varepsilon}\right)$ and of the solution, that is to say that we need to provide a mesh (cartesian for FD, or simplicial for FE) whose space step $h$ satisfies

$$
h \ll \varepsilon .
$$

For very small values of $\varepsilon$ it is not realistic to mesh the domain and assemble the discretized version of (6.1).

The classical approach to solve this difficulty consists in using the homogenization theory and to solve the associated homogenized problem. Nevertheless, what is the error that we obtain for such a numerical solution? Moreover, are there other alternatives? We try to give answers to these questions in the following. We start by recalling the classical error estimates that are obtained when one applies the finite element method to classical elliptic problems, and apply the results to get an estimation of the error obtained for the numerical solution of the homogenized problem. We afterwards explain the method of multiscale finite elements of Hou et al. [10].

\subsection{Classical error estimates}

The classical method to find error estimates for the finite element method is based on 2 ingredients:

- An abstract lemma (Céa's lemma or Strang's lemma) which links the approximation error to the interpolation error.

- A general result which typically depends on the considered finite element which expresses the behavior of the interpolation error depending on the mesh step $h$ and the regularity of the exact solution.

We detail these two aspects hereafter on a model problem. 


\subsubsection{The model problem}

We consider in what follows the homogeneous Dirichlet problem on a bounded regular domain $\Omega$. In order to simplify the exposition, we further assume that $\Omega \subset \mathbb{R}^{2}$, although most (if not all) of what we present here extends naturally to dimension 3 or higher.

The Dirichlet problem with non constant coefficients we have in mind consists in solving

$$
\left\{\begin{array}{l}
-\operatorname{div}(\omega(x) \nabla u)=f \text { in } \Omega, \\
u=0 \text { on } \partial \Omega .
\end{array}\right.
$$

It is well known that it has a variational formulation that reads

$$
\text { Find } u \in H_{0}^{1}(\Omega) \text { such that } \forall v \in H_{0}^{1}(\Omega), \int_{\Omega} \omega(x) \nabla u(x) \cdot \nabla v(x) d x=\int_{\Omega} f(x) v(x) d x .
$$

We also assume that there exist $\alpha>\beta>0$ such that the rigidity matrix $\omega$ satisfies

$$
\forall x \in \Omega, \alpha \operatorname{Id} \geq \omega(x) \geq \beta \text { Id. }
$$

Furthermore, we assume that the coefficients of $\omega$ are regular enough. For the sake of simplicity, we make the assumption that $\omega \in \mathcal{C}^{\infty}(\Omega)$. Usually, the proof of the existence of a solution to (6.3) relies on the Lax-Milgram Theorem. To be more specific, let us consider an abstract framework for the problem. Calling $V=H_{0}^{1}(\Omega)$, the variational formulation can be written as

$$
\text { Find } u \in V \text { such that } \forall v \in V, a(u, v)=l(v)
$$

where $a$ is a bilinear form on a Hilbert space $V$, and $l$ a linear form defined also on $V$, namely

$$
\begin{aligned}
a(u, v) & =\int_{\Omega} \omega(x) \nabla u(x) \cdot \nabla v(x) d x, \\
l(v) & =\int_{\Omega} f(x) v(x) d x .
\end{aligned}
$$

If $l$ is continuous and $a$ is continuous and $V$-coercive, it is well known that the Lax-Milgram theorem applies and there exists a unique solution $u$ of the problem (6.4).

A finite element approximation of (6.3) consists in building a finite dimensional subspace $V_{h}$ of $V$ and to look for a solution of the discrete variational formulation

$$
\text { Find } u_{h} \in V_{h} \text { such that } \forall v_{h} \in V_{h}, a\left(u_{h}, v_{h}\right)=l\left(v_{h}\right) .
$$

Of course, since $V_{h}$ is also a Hilbert space, the Lax-Milgram Theorem still applies on $V_{h}$ and (6.5) also possesses a unique solution $u_{h}$ usually called the approximate solution.

\subsubsection{Céa's and Strang's lemmas}

The error between $u$ and $u_{h}$ is evaluated thanks to Céa's lemma.

Lemma 6.1 (Céa's lemma). With the above hypotheses, one has

$$
\exists C>0 \text { such that }\left\|u-u_{h}\right\|_{V} \leq C \inf _{w_{h} \in V_{h}}\left\|u-w_{h}\right\|_{V} .
$$

Proof. Since $a$ is coercive on $V$, there exists $\alpha>0$, such that

$$
\alpha\left\|u-u_{h}\right\|_{V}^{2} \leq a\left(u-u_{h}, u-u_{h}\right) .
$$

Now, since $u$ solves (6.4) and $u_{h}$ solves (6.5), using $v=v_{h}=u_{h}-w_{h}$ as a test function leads to

$$
a\left(u, u_{h}-w_{h}\right)=l\left(u_{h}-w_{h}\right)=a\left(u_{h}, u_{h}-w_{h}\right),
$$

which gives

$$
a\left(u-u_{h}, u_{h}-w_{h}\right)=0 .
$$

Using this in (6.7) allows us to write

$$
\begin{aligned}
\alpha\left\|u-u_{h}\right\|_{V}^{2} & \leq a\left(u-u_{h}, u-u_{h}\right) \\
& =a\left(u-u_{h}, u-u_{h}+u_{h}-w_{h}\right) \\
& =a\left(u-u_{h}, u-w_{h}\right) \\
& \leq M\left\|u-u_{h}\right\|_{V}\left\|u-w_{h}\right\|_{V}
\end{aligned}
$$


from the continuity of $a$. Dividing both terms by $\left\|u-u_{h}\right\|_{V}$ gives

$$
\left\|u-u_{h}\right\|_{V} \leq \frac{M}{\alpha}\left\|u-w_{h}\right\|_{V}
$$

which leads to (6.6) since $w_{h}$ is arbitrary in $V_{h}$.

Céa's lemma plays a prominent role in the error estimation between the exact and approximate solutions of elliptic problems since it links the approximation error $\left\|u-u_{h}\right\|_{V}$ made by solving the problem on a subspace $V_{h} \subset V$ to the interpolation error $\inf _{w_{h} \in V_{h}}\left\|u-w_{h}\right\|_{V}$. Notice that this latter does not depend on the problem, but only on the way the exact solution $u$ is close to $V_{h}$, or in other words, on how well $u$ can be interpolated on $V_{h}$.

As we shall see, for the error estimation on the homogenized problem, one has to face the problem that the exact and approximate problems are no longer the same. The approximate variational formulation becomes

$$
\text { Find } u_{h} \in V_{h} \text { such that } \forall v_{h} \in V_{h}, a_{h}\left(u_{h}, v_{h}\right)=l_{h}\left(v_{h}\right) \text {. }
$$

In that case, there exists a natural extension of Céa's lemma which is known as Strang's lemma.

Lemma 6.2 (Strang's lemma). Assume that $l_{h}$ is uniformly continuous on $V$ and $a_{h}$ is uniformly continuous and coercive on $V$ then one has

$$
\left\|u-u_{h}\right\|_{V} \lesssim\left(\inf _{w_{h} \in V_{h}}\left\|u-w_{h}\right\|_{V}+\sup _{v_{h} \in V_{h}} \frac{\left|a_{h}\left(u, v_{h}\right)-l_{h}\left(v_{h}\right)\right|}{\left\|v_{h}\right\|_{V}}\right) .
$$

Proof. Since $a_{h}$ is uniformly coercive on $V$, there exists $\alpha>0$, such that

$$
\alpha\left\|u_{h}-w_{h}\right\|_{V}^{2} \leq a_{h}\left(u_{h}-w_{h}, u_{h}-w_{h}\right) .
$$

Now, since $u_{h}$ solves (6.8), using $v_{h}=u_{h}-w_{h}$ as a test function leads to

$$
\begin{aligned}
a_{h}\left(u_{h}-w_{h}, u_{h}-w_{h}\right)= & a_{h}\left(u-w_{h}, u_{h}-w_{h}\right) \\
& -\left(a_{h}\left(u, u_{h}-w_{h}\right)-a_{h}\left(u_{h}, u_{h}-w_{h}\right)\right) \\
\leq & M\left\|u-w_{h}\right\|_{V}\left\|u_{h}-w_{h}\right\|_{V} \\
& +\left|a_{h}\left(u, u_{h}-w_{h}\right)-l_{h}\left(u_{h}-w_{h}\right)\right|
\end{aligned}
$$

where we have used that $a_{h}$ is uniformly continuous on $V$. This gives, using (6.10), and dividing by $\left\|u_{h}-w_{h}\right\|_{V}$

$$
\left\|u_{h}-w_{h}\right\|_{V} \leq \frac{M}{\alpha}\left\|u-w_{h}\right\|_{V}+\frac{1}{\alpha} \frac{\left|a_{h}\left(u, u_{h}-w_{h}\right)-l_{h}\left(u_{h}-w_{h}\right)\right|}{\left\|u_{h}-w_{h}\right\|_{V}},
$$

which leads to

$$
\left\|u_{h}-w_{h}\right\|_{V} \leq \frac{M}{\alpha}\left\|u-w_{h}\right\|_{V}+\frac{1}{\alpha} \inf _{v_{h} \in V_{h}} \frac{\left|a_{h}\left(u, v_{h}\right)-l_{h}\left(v_{h}\right)\right|}{\left\|v_{h}\right\|_{V}} .
$$

Eventually, the triangle inequality $\left\|u-u_{h}\right\|_{V} \leq\left\|u-w_{h}\right\|_{V}+\left\|u_{h}-w_{h}\right\|_{V}$ leads to (6.9) by taking $C=\max \left\{\frac{M}{\alpha}+1, \frac{1}{\alpha}\right\}$.

Compared to Céa's lemma, Strang's lemma measures not only the interpolation error, but also how well the discretized problem approaches the exact one. Indeed, this latter term would vanish if one replaces $a_{h}$ and $l_{h}$ by $a$ and $l$ respectively.

\subsubsection{Regularity}

The regularity of the solution of (6.2) is a topic outside the scope of these notes. Without entering into details, let us just mention that, up to now, the solution that has been built has only a $H^{1}$ regularity (all derivatives of $u$ are square integrable on $\Omega$ ). This regularity can be enhanced depending on the regularity of the right hand side $f$. We just mention the following classical regularity result.

Theorem 6.1. Assume that $\Omega$ is convex or smooth ${ }^{2}$. Then if $f \in H^{k}(\Omega)$, the solution $u$ of (6.2) belongs to $H^{k+2}(\Omega)$ and, moreover,

$$
\exists C(\omega, \Omega)>0 \text { such that }\|u\|_{H^{k+2}} \leq C(\omega, \Omega)\|f\|_{H^{k}} .
$$

We refer the interested reader to [7] where a proof is given. Notice that we have given the general regularity result (for all $k$ ) although we mainly use in practive this result for $k=0$, namely

$$
f \in L^{2}(\Omega) \Rightarrow u \in H^{2}(\Omega) .
$$

${ }^{2}$ By this we mean $C^{\infty}$. This can be improved by lowering the regularity to $\mathrm{C}^{k+2}$. 
As we shall see in the next section, this regularity property plays a very important role in the error estimation for the finite element method.

\subsubsection{Interpolation error with Lagrange's finite elements}

Eventually to complete this section, we recall without proof the classical estimation of the interpolation error when one uses the conformal finite element $P^{k}$ method with polynomials of degree $k \geq 1$ on triangular conformal meshes.

Proposition 6.1. Assume $\Omega$ is a bounded polyhedral domain of $\mathbb{R}^{2}$ meshed with a regular family of triangulations $\mathcal{T}_{h}$. Take $u \in H^{k+1}(\Omega)$, and $V_{h}=P^{k}\left(\mathcal{T}_{h}\right)$. Then one has the estimation

$$
\forall h>0,\left\|u-I_{h}(u)\right\|_{H^{l}(\Omega)} \leq C h^{k+1-l}|u|_{H^{k+1}(\Omega)}
$$

for all $l \leq k+1$.

In the preceding lemma, we have called $I_{h}: H^{2}(\Omega) \rightarrow P^{k}\left(\mathcal{T}_{h}\right)$ the interpolation operator. For the proof of this result, we refer the reader to classical textbooks on finite elements like [3] or [6]. We nevertheless give a few hints. The main idea is to prove the same kind of estimation on a reference simplex $\hat{K}$

$$
\exists C>0, \forall \hat{u} \in H^{k+1}(\hat{K}),\left\|\hat{u}-I_{h}(\hat{u})\right\|_{H^{k+1}(\hat{K})} \leq C|\hat{u}|_{H^{k+1}(\hat{K})} \cdot
$$

(This is usually done by showing that the two norms $\|\cdot\|_{H^{k+1}(\hat{K})}$, and $|\cdot|_{H^{k+1}(\hat{K})}+\left\|I_{h}(\cdot)\right\|_{H^{k+1}(\hat{K})}$ are actually equivalent on $H^{k+1}(\hat{K})$. Applying this to $\hat{u}-I_{h}(\hat{u})$ leads to the desired inequality.) It then follows that for all $l \leq k+1$,

$$
\exists C>0, \forall \hat{u} \in H^{k+1}(\hat{K}),\left\|\hat{u}-I_{h}(\hat{u})\right\|_{H^{l}(\hat{K})} \leq C|\hat{u}|_{H^{k+1}(\hat{K})},
$$

where the first norm is now the $H^{l}$ norm. Eventually, one has to see that both terms do not scale identically. More precisely, calling $u$ the map defined by $u(x)=\hat{u}(h x), \forall x \in \hat{K}$, one has

$$
\begin{aligned}
\left|\hat{u}-I_{h}(\hat{u})\right|_{H^{l}(h \hat{K})} & =h^{1-l}\left|\hat{u}-I_{h}(\hat{u})\right|_{H^{l}(\hat{K})} \\
& \leq C h^{1-l}|\hat{u}|_{H^{k+1}(\hat{K})} \\
& =C h^{k+1-l}|u|_{H^{k+1}(h \hat{K})} .
\end{aligned}
$$

Summing the obtained inequality (after having squared it) on all the simplices of the triangulation and on all $0 \leq l \leq k$ leads to the desired result.

Remark 6.1. Notice that the preceding interpolation inequality leads to an estimation of the $L^{2}$ norm

$$
\exists C>0, \forall u \in H^{k+1}(\Omega),\left\|u-I_{h}(u)\right\|_{L^{2}} \leq C h^{k+1}|u|_{H^{k+1}} .
$$

From this interpolation theory together with Céa's (or Strang's) lemma, one can estimate the error between the exact and approximate solutions.

Theorem 6.2. Let $f \in H^{k-1}(\Omega)$. Then the finite element solution $u_{h}$ satisfies the error estimate

$$
\left\|u-u_{h}\right\|_{H^{1}} \lesssim h^{k}\|f\|_{H^{k-1}} .
$$

In particular taking $k=1$ and $P^{1}$ finite elements leads to the estimate

$$
\left\|u-u_{h}\right\|_{H^{1}} \lesssim h\|f\|_{L^{2}} .
$$

Proof. From the regularity result, we know that $u \in H^{k+1}(\Omega)$. Then, using Céa's lemma, we get

$$
\begin{aligned}
\left\|u-u_{h}\right\|_{H^{1}} & \lesssim \inf _{w_{h} \in V_{h}}\left\|u-w_{h}\right\|_{H^{1}} \\
& \lesssim h^{k}|u|_{H^{k+1}} \\
& \lesssim h^{k}\|f\|_{H^{k-1}} .
\end{aligned}
$$

Remark 6.2. Notice that the degree of the Lagrange finite element used is intimately linked to the regularity of the exact solution $u$ to the problem. Indeed, the estimation given before is useless if $f \notin H^{k-1}$. In that case, this means that using $P^{k}$ finite element is useless. For instance, if $f \in L^{2}$ but $f \notin H^{1}$, then the optimal error is obtained using the $P^{1}$ finite element method.

Notice that so far we have only estimated the $H^{1}$ distance between the exact and approximate solutions. Although a better estimate exists for the $L^{2}$ norm for interpolation, Céa's lemma is a priori wrong when dealing with $L^{2}$ norms. We end this section with the so-called Aubin-Nitsche's lemma which permits us to estimate the $L^{2}$ norm error between the solution to the exact problem $u$ and the approximate solution $u_{h}$.

Lemma 6.3 (Aubin-Nitsche). Assume that $u \in H^{k+1}(\Omega)$. Then, 


$$
\exists C>0,\left\|u-u_{h}\right\|_{L^{2}} \leq C h^{k+1}|u|_{H^{k+1}} .
$$

Proof. We start by noticing that

$$
\left\|u-u_{h}\right\|_{L^{2}}=\sup _{g \in L^{2}} \frac{\int_{\Omega}\left(u-u_{h}\right) g d x}{\|g\|_{L^{2}}} .
$$

Now, we introduce the (unique) solution $v_{g}$ to the problem

$$
\text { Find } v \in V \text {, such that } a\left(w, v_{g}\right)=\int_{\Omega} g w d x \text {. }
$$

Notice that this is not the same problem as before since the unknown is in the right hand side of $a$. More precisely, it is easily seen that $v_{g}$ solves

$$
\left\{\begin{array}{l}
-\operatorname{div}\left(\omega^{T}(x) \nabla v_{g}\right)=g \text { in } \Omega, \\
u=0 \text { on } \partial \Omega .
\end{array}\right.
$$

In particular, the regularity Theorem 6.1 applies and $g \in L^{2}(\Omega) \Rightarrow v_{g} \in H^{2}(\Omega)$ and more precisely,

$$
\left\|v_{g}\right\|_{H^{2}} \leq C\|g\|_{L^{2}} .
$$

Inserting $w=u-u_{h}$ leads to

$$
\begin{aligned}
\int_{\Omega}\left(u-u_{h}\right) g d x & =a\left(u-u_{h}, v_{g}\right) \\
& =a\left(u-u_{h}, v_{g}-w_{h}\right)
\end{aligned}
$$

for all $w_{h} \in V_{h}$, since we know that $a\left(u-u_{h}, w_{h}\right)=0$. But, since $v_{g} \in V$, we have (taking for instance $w_{h}=I_{h}\left(v_{g}\right)$ ),

$$
\begin{aligned}
\left|a\left(u-u_{h}, v_{g}-I_{h}\left(v_{g}\right)\right)\right| & \lesssim\left\|u-u_{h}\right\|_{V}\left\|v_{g}-I_{h}\left(v_{g}\right)\right\|_{V} \\
& \lesssim h^{k+1}|u|_{H^{k+1}}\left|v_{g}\right|_{H^{2}} \\
& \lesssim h^{k+1}|u|_{H^{k+1}}\|g\|_{L^{2}},
\end{aligned}
$$

in view of (6.12). Therefore we get

$$
\int_{\Omega}\left(u-u_{h}\right) g d x \lesssim h^{k+1}|u|_{H^{k+1}}\|g\|_{L^{2}},
$$

and

$$
\begin{aligned}
\left\|u-u_{h}\right\|_{L^{2}} & =\sup _{g \in L^{2}} \frac{\int_{\Omega}\left(u-u_{h}\right) g d x}{\|g\|_{L^{2}}} \\
& \lesssim h^{k+1}|u|_{H^{k+1}}
\end{aligned}
$$

which proves the lemma.

Remark 6.3. For the Aubin-Nitsche's lemma, the hypotheses are those of Céa's lemma. In other words, the discrete and continuous problems should be the same. In that case, we indeed get an improvement for the approximation of the $L^{2}$ norm compared to the $H^{1}$ norm. When instead we are under the hypotheses of Strang's lemma (that is to say the discrete and continuous problems are no longer the same), we can not rely on Aubin-Nitsche's lemma and have no better estimate for the $L^{2}$ norm.

\subsection{Application to homogenization problems}

We now turn to the application of the preceding results to the homogenized model problem (6.1). We recall that homogenization theory provides an expansion of the solution $u$ to (6.1) as

$$
u(x)=u_{0}(x)+\varepsilon u_{1}\left(x, \frac{x}{\varepsilon}\right)+\cdots
$$

in $H^{1}(\Omega)$, where $u_{0}$ solves the homogenized problem ${ }^{3}$

\footnotetext{
${ }^{3}$ For the sake of simplicity of notation, we here have denoted by $\bar{A}$ what was called $A_{\text {eff }}$ in the preceding chapters. This will prove more efficient when we will have to compute the entries of the tensor, or more precisely of its discretized version.
} 


$$
\left\{\begin{array}{l}
-\operatorname{div}\left(\bar{A} \nabla u_{0}\right)=f \text { in } \Omega, \\
u_{0}=0 \text { on } \partial \Omega,
\end{array}\right.
$$

and

$$
\forall(x, y) \in \Omega \times Y, u_{1}(x, y)=\sum_{i=1}^{2} \frac{\partial u_{0}}{\partial x_{i}}(x) w_{i}(y)
$$

and $w_{i}$ are the correctors, solutions to

$$
\left\{\begin{array}{l}
-\operatorname{div}_{y}\left(A(y)\left(\nabla w_{i}(y)+e_{i}\right)\right)=0 \text { in } Y, \\
w_{i} \text { is } Y \text {-periodic, } \int_{Y} w_{i}(y) d y=0 .
\end{array}\right.
$$

The homogenized tensor $\bar{A}$ is also given in terms of $A$ and $w_{i}$ by the formula

$$
\begin{aligned}
\bar{A}_{i, j} & =\int_{Y} A(y)\left(e_{i}+\nabla w_{i}\right) \cdot e_{j} \mathrm{~d} y \\
& =\int_{Y} A(y)\left(e_{i}+\nabla w_{i}\right) \cdot\left(e_{j}+\nabla w_{j}\right) \mathrm{d} y .
\end{aligned}
$$

At this level, we have two sources of error:

- The error that one makes when approximating the exact solution $u$ by a multiscale expansion $u(x) \sim u_{0}(x)$ or $u(x) \sim u_{0}(x)+\varepsilon u_{1}\left(x, \frac{x}{\varepsilon}\right)$ or similar expansions. This error is quantified in $L^{2}$ or $H^{1}$ norms and is evaluated in terms of $\varepsilon$;

- The error that one makes when approximating the above terms $\left(u_{0}, u_{1}\right.$, etc.) by their corresponding finite element approximations. These errors are estimated again in $L^{2}$ or $H^{1}$ typically and depend on the mesh size $h$. For this part we need to use the preceding results.

In what follows, we start with the second estimation, and turn to the first afterwards.

\subsubsection{Error estimation of the finite element approximations of $u_{0}$ and $u_{1}$}

When solving the homogenized problem for $u_{0}$, one usually computes an approximate homogenized tensor $\bar{A}_{h}$ by solving the variational problem corresponding to (6.13), that is to say

$$
\text { Find } w_{i} \in H_{\#}^{1}(Y), \forall v \in H_{\#}^{1}(Y), \int_{Y} A(y) \nabla w_{i} \cdot \nabla v \mathrm{~d} y=-\int_{Y} A(y) e_{i} \cdot \nabla v \mathrm{~d} y
$$

and more precisely, its discretized version.

The theory of approximation, just stated in the previous section shows that there is an error estimation, when one solves (6.15) on a regular family of triangulations $\left(\mathcal{T}_{h}\right)_{h>0}$ and using the $P^{k}$ finite elements. Namely, one has

$$
\left\|w_{i}-w_{h, i}\right\|_{H^{1}(\Omega)} \leq C h^{k}\left|w_{i}\right|_{H^{k+1}(\Omega)}
$$

provided that $w_{i} \in H^{k+1}(Y)$. Since the coefficients of the tensor $A$ are assumed to be smooth, it is easily seen that the correctors $w_{i}$ are regular, in the sense that $w_{i} \in \mathcal{C}^{\infty}(Y)$. Using a $P^{k}$ finite element method thus leads to

$$
\left\|w_{i}-w_{h, i}\right\|_{H^{1}(\Omega)} \leq C h^{k} .
$$

Moreover, while computing the homogenized tensor $\bar{A}$, one therefore makes an error which can be estimated on each coefficient

$$
\begin{aligned}
\left|\bar{A}_{i, j}-\bar{A}_{h ; i, j}\right| & =\left|\int_{Y}\left(A(y)\left(e_{i}+\nabla w_{i}\right) \cdot e_{j}-A(y)\left(e_{i}+\nabla w_{h, i}\right) \cdot e_{j}\right) \mathrm{d} y\right| \\
& \lesssim \int_{Y}\left|A(y)\left(\nabla\left(w_{i}-w_{h, i}\right)\right) \cdot e_{j} \mathrm{~d} y\right| \\
& \lesssim\left\|w_{i}-w_{h, i}\right\|_{H^{1}} \\
& \lesssim h^{k} .
\end{aligned}
$$

Of course, for less regular coefficients $A(y)$, one gets lower order errors in the computation of $A$.

Remark 6.4. We stress the fact that since $Y$ is a unit cube, it is easy to have a regular family of triangulations of $Y$. It suffices to divide $Y$ into small squares of edgelength $h$, and to further subdivide those small squares into triangles.

We now turn to the problem of the approximation of $u_{0}$. In our finite element setting, we have to estimate the error 
between the solution to (6.13) and the corresponding approximate problem. With this aim, we write the variational formulation of (6.13), namely, setting $V=H_{0}^{1}(\Omega)$

$$
\text { Find } u_{0} \in V, \forall v \in V, \int_{\Omega} \bar{A} \nabla u_{0}(y) \cdot \nabla v(y) \mathrm{d} y=\int_{\Omega} f(y) v(y) \mathrm{d} y,
$$

the discrete variational formulation becomes

$$
\text { Find } u_{0, h} \in V_{h}, \forall v_{h} \in V_{h}, \int_{\Omega} \bar{A}_{h} \nabla u_{0, h}(y) \cdot \nabla v_{h}(y) \mathrm{d} y=\int_{\Omega} f(y) v_{h}(y) \mathrm{d} y .
$$

and the error estimation reads as follows.

Lemma 6.4. Let $\left(\mathcal{T}_{h}\right)_{h}$ be a regular family of triangulations of $\Omega$. Assume that there exists $k \geq 1$ such that $f \in$ $H^{k-1}(\Omega)$ and that we use the $P^{k}$ Lagrange finite element method to compute numerically $u_{0}$. Then

$$
\left\|u_{0}-u_{0, h}\right\|_{H^{1}} \lesssim h^{k}\|f\|_{H^{k-1}}
$$

Proof. We are exactly in a position to apply Strang's lemma. Indeed, we set

$$
\begin{aligned}
\forall u, v \in V, a(u, v) & =\int_{\Omega} \bar{A} \nabla u(y) \cdot \nabla v(y) \mathrm{d} y, \\
\forall u_{h}, v_{h} \in V_{h}, a_{h}\left(u_{h}, v_{h}\right) & =\int_{\Omega} \bar{A}_{h} \nabla u_{h}(y) \cdot \nabla v_{h}(y) \mathrm{d} y, \\
\forall v \in V, l(v)=l_{h}(v) & =\int_{\Omega} f(y) v(y) \mathrm{d} y .
\end{aligned}
$$

Then, applying Strang's lemma, we get the estimate

$$
\left\|u_{0}-u_{0, h}\right\|_{V} \leq C\left(\inf _{w_{h} \in V_{h}}\left\|u_{0}-w_{h}\right\|_{V}+\sup _{v_{h} \in V_{h}} \frac{\left|a_{h}\left(u_{0}, v_{h}\right)-l_{h}\left(v_{h}\right)\right|}{\left\|v_{h}\right\|_{V}}\right) .
$$

The first term of the right hand side follows classical interpolation estimation while for the second, we have

$$
\begin{aligned}
a_{h}\left(u_{0}, v_{h}\right)-l_{h}\left(v_{h}\right) & =\int_{\Omega} \bar{A}_{h} \nabla u_{0}(y) \cdot \nabla v_{h}(y) \mathrm{d} y-\int_{\Omega} f(y) v_{h}(y) \mathrm{d} y \\
& =\int_{\Omega}\left(\bar{A}_{h}-\bar{A}\right) \nabla u_{0}(y) \cdot \nabla v_{h}(y) \mathrm{d} y
\end{aligned}
$$

and therefore

$$
\left|a_{h}\left(u_{0}, v_{h}\right)-l_{h}\left(v_{h}\right)\right| \leq\left\|\bar{A}_{h}-\bar{A}\right\|_{\infty}\left\|u_{0}\right\|_{V}\left\|v_{h}\right\|_{V}
$$

Putting this in (6.19), we get

$$
\begin{aligned}
\left\|u_{0}-u_{0, h}\right\|_{V} & \leq C\left(\inf _{w_{h} \in V_{h}}\left\|u_{0}-w_{h}\right\|_{V}+\left\|\bar{A}_{h}-\bar{A}\right\|_{\infty}\left\|u_{0}\right\|_{V}\right) \\
& \leq C h^{k}\left\|u_{0}\right\|_{H^{k+1}}
\end{aligned}
$$

using (6.16) and the classical $P^{k}$ finite element estimation.

Remark 6.5. We stress the fact that we get a classical error estimation although we need to solve an auxiliary problem. It is important to realize that the preceding estimate contains two terms. The first one which behaves classically as for the usual elliptic problems and the second one which contains the estimation of the auxiliary problem. In particular, in order to get the estimate, we have assumed that the mesh on $Y$ that was used to solve the cell problem has a space step $h$ comparable to the one that is used to solve the homogenized problem. Moreover, the degree of the finite element used is also important. It is easily seen and understandable that in order to get a higher order convergence, one needs to use higher order finite elements not only for the homogenized problem, but also for the cell problem, in order to get a better approximation of the homogenized tensor $\bar{A}$.

Remark 6.6. It is also interesting to notice that, having no equivalent of Aubin-Nitsche's trick (because $\bar{A} \neq \overline{A_{h}}$ ), we do not have a better estimate for the $L^{2}$ norm.

It remains now to estimate the error between the solution $u_{\varepsilon}$ to the original problem and the one built before.

\subsubsection{Error analysis of the multiscale expansion}

In this section we detail the results that were obtained by different authors. Most of the material exposed here can be found in [12]. We remark that $u_{0}$ can only be a good approximation of $u_{\varepsilon}$ in $L^{2}$ but certainly not in $H^{1}$. Indeed, we know from the classical theory, that $u_{\varepsilon} \rightarrow u_{0}$ weakly in $H^{1}$ and strongly in $L^{2}$. Moreover, the 2 scale homogenization gives more information, $\left(\left(u_{\varepsilon}\right)_{\varepsilon}\right.$ and $\left(\nabla u_{\varepsilon}\right)_{\varepsilon}$ being uniformly bounded in $\left.L^{2}\right)$ namely, we know that 


$$
\begin{aligned}
u_{\varepsilon} & \rightarrow u_{0}, \\
\nabla u_{\varepsilon} & \rightarrow \nabla_{x} u_{0}+\nabla_{y} u_{1} .
\end{aligned}
$$

Therefore, although $\left\|u_{\varepsilon}-u_{0}\right\|_{L^{2}}$ tends to 0 , we do not have that $\left\|\nabla u_{\varepsilon}-\nabla u_{0}\right\|_{L^{2}}$ tends to 0 (since $\nabla u_{\varepsilon}-\nabla u_{0} 2$-scale converges to $\left.\nabla_{y} u_{1}\right)$. As far as the $H^{1}$ norm is concerned we know that

$$
u_{\varepsilon}(x)-u_{0}(x)-\varepsilon u_{1}\left(x, \frac{x}{\varepsilon}\right) \rightarrow 0
$$

strongly in $H^{1}$. This gives another way to understand the preceding remarks, since we get

$$
\begin{aligned}
\varepsilon u_{1}\left(x, \frac{x}{\varepsilon}\right) & \rightarrow 0 \text { in } L^{2} \\
\nabla\left(\varepsilon u_{1}\left(x, \frac{x}{\varepsilon}\right)\right)=\varepsilon \nabla_{x} u_{1}\left(x, \frac{x}{\varepsilon}\right)+\nabla_{y} u_{1}\left(x, \frac{x}{\varepsilon}\right) & \rightarrow \int_{Y} \nabla_{y} u_{1}(x, y) d y \text { weakly in } L^{2} .
\end{aligned}
$$

Again, although $\int_{Y} \nabla_{y} u_{1}(x, y) d y=0$ (since $u_{1}$ is a $y$-periodic function) the convergence is only weak, and it is easily seen that

$$
\left\|\nabla\left(\varepsilon u_{1}\left(x, \frac{x}{\varepsilon}\right)\right)\right\|_{L^{2}} \rightarrow\left\|\nabla_{y} u_{1}(x, y)\right\|_{L^{2}(\Omega \times Y)} \neq 0 \text { as } \varepsilon \rightarrow 0 .
$$

Moreover, the preceding remarks are not quantitative. In particular, we do not know the behavior (say in terms of powers of $\varepsilon$ ) of these convergences. The theorems of this section give this behavior.

Theorem 6.3. We have

$$
\left\|u_{\varepsilon}(x)-u_{0}(x)-\varepsilon u_{1}\left(x, \frac{x}{\varepsilon}\right)\right\|_{H^{1}} \lesssim \sqrt{\varepsilon} .
$$

Corollary 6.1. We have

$$
\left\|u_{\varepsilon}(x)-u_{0}(x)\right\|_{L^{2}} \lesssim \varepsilon
$$

Theorem 6.3 as well as Corollary 6.1 are consequences of a much more difficult and precise theorem given below. In order to explain it, let us remark that the approximation $u_{0}(x)-\varepsilon u_{1}\left(x, \frac{x}{\varepsilon}\right)$ does not satisfy the Dirichlet boundary conditions on $\partial \Omega$. Indeed, there is no reason that $u_{1}$ should vanish on the boundary. The idea is therefore to introduce a corrector to the boundary condition. Namely, we introduce $\theta_{\varepsilon}$ solution to

$$
\left\{\begin{array}{l}
-\operatorname{div}\left(A\left(\frac{x}{\varepsilon}\right) \nabla \theta_{\varepsilon}\right)=0 \text { in } \Omega, \\
\theta_{\varepsilon}=u_{1}\left(x, \frac{x}{\epsilon}\right) \text { on } \partial \Omega .
\end{array}\right.
$$

It is now clear that

$$
u_{\varepsilon}(x)-u_{0}(x)-\varepsilon\left(u_{1}\left(x, \frac{x}{\varepsilon}\right)-\theta_{\varepsilon}(x)\right)=0 \text { on } \partial \Omega .
$$

We are now in a position to state the theorem.

Theorem 6.4. Suppose that $u_{0} \in H^{2}$. Then

$$
\left\|u_{\varepsilon}(x)-u_{0}(x)-\varepsilon\left(u_{1}\left(x, \frac{x}{\varepsilon}\right)-\theta_{\varepsilon}(x)\right)\right\|_{H^{1}} \lesssim \varepsilon\left\|u_{0}\right\|_{H^{2}} .
$$

Proof. As we shall see, the proof is rather complicated and follows several steps. We first rewrite the problem as

$$
\begin{aligned}
v_{\varepsilon} & =A\left(\frac{x}{\varepsilon}\right) \nabla u_{\varepsilon}, \\
-\operatorname{div}\left(v_{\varepsilon}\right) & =f .
\end{aligned}
$$

Making a (formal) multiscale expansion of the preceding equations, we get

$$
u_{\varepsilon}=u_{0}\left(x, \frac{x}{\varepsilon}\right)+\varepsilon u_{1}\left(x, \frac{x}{\varepsilon}\right)+\cdots
$$




$$
v_{\varepsilon}=v_{0}\left(x, \frac{x}{\varepsilon}\right)+\varepsilon v_{1}\left(x, \frac{x}{\varepsilon}\right)+\cdots
$$

Putting these expansions in the preceding equations, and equating the terms with the same power in $\varepsilon$, we get

$$
\begin{aligned}
A(y) \nabla_{y} u_{0} & =0, \\
-\operatorname{div}_{y} v_{0} & =0, \\
A(y)\left(\nabla_{y} u_{1}+\nabla_{x} u_{0}\right) & =v_{0}, \\
-\operatorname{div}_{y} v_{1}-\operatorname{div}_{x} v_{0} & =f .
\end{aligned}
$$

Equation (6.22) gives as usual the fact that $u_{0}$ does not depend on $y$, while (6.23) and (6.24) together give the cell problem. The homogenized problem is usually obtained by integrating (6.25) over the cell $Y$.

Guided by this computation, we thus define

$$
v_{0}(x, y)=A(y)\left(\nabla_{x} u_{0}(x)+\nabla_{y} u_{1}(x, y)\right) .
$$

Since $u_{0}$ and $u_{1}$ are linked by the cell problem, we know that $\operatorname{div}_{y} v_{0}=0$. On the other hand, we also know (since everything depends only on the slow variable $x$ ) that $\operatorname{div}_{y} \bar{A} \nabla u_{0}=0$. Therefore, $\operatorname{div}_{y}\left(v_{0}-\bar{A} \nabla u_{0}\right)=0$, and since we are in dimension 2 , there exists $q(x, y)$ such that

$$
v_{0}-\bar{A} \nabla u_{0}=\nabla_{y}^{\perp} q(x, y) .
$$

Here, we have denoted by $\nabla_{y}^{\perp}=\left(-\frac{\partial}{\partial y_{2}}, \frac{\partial}{\partial y_{1}}\right)$ the $2 \mathrm{D}$ curl. It is easily seen that $q$ is $Y$-periodic and depends linearly on $\nabla_{x} u_{0}$, so that one has the estimate

$$
\sup _{y \in Y}\left|\nabla_{x} q(x, y)\right| \lesssim \sum_{i, j}\left|\frac{\partial^{2} u_{0}}{\partial x_{i} \partial x_{j}}(x)\right|, \text { a.e. in } \Omega \text {. }
$$

We now set $v_{1}(x, y)=\nabla_{x}^{\perp} q(x, y)$, and notice that

$$
\begin{aligned}
\operatorname{div}_{y} v_{1} & =\operatorname{div}_{y} \nabla_{x}^{\perp} q \\
& =-\frac{\partial^{2} q}{\partial y_{1} \partial x_{2}}+\frac{\partial^{2} q}{\partial y_{2} \partial x_{1}} \\
& =-\operatorname{div}_{x} \nabla_{y}^{\perp} q \\
& =-\operatorname{div}_{x}\left(v_{0}-\bar{A} \nabla u_{0}\right) \\
& =-\operatorname{div}_{x} v_{0}-f
\end{aligned}
$$

so that we recover (6.25).

We also have (due to (6.26)) that

$$
\sup _{y \in Y}\left|v_{1}(x, y)\right| \lesssim \sum_{i, j}\left|\frac{\partial^{2} u_{0}}{\partial x_{i} \partial x_{j}}(x)\right|, \text { a.e. } x \in \Omega
$$

and from the definition of $v_{1}, \operatorname{div}_{x} v_{1}(x, y)=0$ in $\Omega \times Y$.

Now, we set

$$
\begin{aligned}
& z_{\varepsilon}(x)=u_{\varepsilon}(x)-u_{0}(x)-\varepsilon u_{1}\left(x, \frac{x}{\varepsilon}\right) \\
& \eta_{\varepsilon}(x)=A\left(\frac{x}{\varepsilon}\right) \nabla u_{\varepsilon}(x)-v_{0}\left(x, \frac{x}{\varepsilon}\right)-\varepsilon v_{1}\left(x, \frac{x}{\varepsilon}\right)
\end{aligned}
$$

and we compute

$$
\begin{aligned}
A\left(\frac{x}{\varepsilon}\right) \nabla z_{\varepsilon}(x)-\eta_{\varepsilon}(x)= & -A\left(\frac{x}{\varepsilon}\right)\left(\nabla u_{0}(x)+\nabla_{y} u_{1}\left(x, \frac{x}{\varepsilon}\right)\right) \\
& -\varepsilon A\left(\frac{x}{\varepsilon}\right) \nabla_{x} u_{1}\left(x, \frac{x}{\varepsilon}\right)+v_{0}\left(x, \frac{x}{\varepsilon}\right)+\varepsilon v_{1}\left(x, \frac{x}{\varepsilon}\right) \\
= & \varepsilon\left(v_{1}\left(x, \frac{x}{\varepsilon}\right)-A\left(\frac{x}{\varepsilon}\right) \nabla u_{1}\left(x, \frac{x}{\varepsilon}\right)\right) .
\end{aligned}
$$

Using (6.28), we therefore get

$$
\left\|A\left(\frac{x}{\varepsilon}\right) \nabla z_{\varepsilon}(x)-\eta_{\varepsilon}(x)\right\|_{L^{2}} \lesssim \varepsilon\left\|u_{0}\right\|_{H^{2}},
$$

while 


$$
\begin{aligned}
\operatorname{div} \eta_{\varepsilon}(x)= & \operatorname{div}\left(A\left(\frac{x}{\varepsilon}\right) \nabla u_{\varepsilon}(x)-v_{0}\left(x, \frac{x}{\varepsilon}\right)-\varepsilon v_{1}\left(x, \frac{x}{\varepsilon}\right)\right) \\
= & -f(x)-\operatorname{div}_{x} v_{0}\left(x, \frac{x}{\varepsilon}\right)-\varepsilon^{-1} \operatorname{div}_{y} v_{0}\left(x, \frac{x}{\varepsilon}\right) \\
& -\varepsilon \operatorname{div}_{x} v_{1}\left(x, \frac{x}{\varepsilon}\right)-\operatorname{div}_{y} v_{1}\left(x, \frac{x}{\varepsilon}\right) \\
= & -f(x)-\operatorname{div}_{x} v_{0}\left(x, \frac{x}{\varepsilon}\right)+0+0-\operatorname{div}_{y} v_{1}\left(x, \frac{x}{\varepsilon}\right) \\
= & 0
\end{aligned}
$$

from (6.27).

Eventually, since $w_{\varepsilon}=z_{\varepsilon}+\varepsilon \theta_{\varepsilon} \in H_{0}^{1}(\Omega)$, one has

$$
\begin{aligned}
\int_{\Omega} A\left(\frac{x}{\varepsilon}\right) \nabla w_{\varepsilon} \cdot \nabla w_{\varepsilon} d x & =\int_{\Omega} A\left(\frac{x}{\varepsilon}\right) \nabla\left(z_{\varepsilon}+\varepsilon \theta_{\varepsilon}\right) \cdot \nabla w_{\varepsilon} d x \\
& =\int_{\Omega} A\left(\frac{x}{\varepsilon}\right) \nabla z_{\varepsilon} \cdot \nabla w_{\varepsilon} d x \\
& =\int_{\Omega}\left(A\left(\frac{x}{\varepsilon}\right) \nabla z_{\varepsilon}-\eta_{\varepsilon}\right) \cdot \nabla w_{\varepsilon} d x+\int_{\Omega} \eta_{\varepsilon} \cdot \nabla w_{\varepsilon} d x \\
& =\int_{\Omega}\left(A\left(\frac{x}{\varepsilon}\right) \nabla z_{\varepsilon}-\eta_{\varepsilon}\right) \cdot \nabla w_{\varepsilon} d x
\end{aligned}
$$

in view of (6.29), and the fact that $w_{\varepsilon} \in H_{0}^{1}(\Omega)$. We deduce from this that

which is the desired result.

$$
\left\|\nabla w_{\varepsilon}\right\|_{L^{2}} \lesssim\left\|A\left(\frac{x}{\varepsilon}\right) \nabla z_{\varepsilon}-\eta_{\varepsilon}\right\|_{L^{2}} \lesssim \varepsilon\left\|u_{0}\right\|_{H^{2}}
$$

Proof of Corollary 6.1. We start with

$$
\left\|u_{\varepsilon}(x)-u_{0}(x)-\varepsilon\left(u_{1}\left(x, \frac{x}{\varepsilon}\right)-\theta_{\varepsilon}(x)\right)\right\|_{H^{1}} \lesssim \varepsilon\left\|u_{0}\right\|_{H^{2}},
$$

from which we deduce, by the Poincaré inequality, that

$$
\left\|u_{\varepsilon}(x)-u_{0}(x)-\varepsilon\left(u_{1}\left(x, \frac{x}{\varepsilon}\right)-\theta_{\varepsilon}(x)\right)\right\|_{L^{2}} \lesssim \varepsilon\left\|u_{0}\right\|_{H^{2}} .
$$

But we have

$$
\left\|u_{1}\left(x, \frac{x}{\varepsilon}\right)\right\|_{L^{2}} \lesssim\left\|u_{0}\right\|_{H^{1}}
$$

and (this is somehow tricky)

$$
\left\|\theta_{\varepsilon}(x)\right\|_{L^{2}} \lesssim\left\|u_{1}\left(x, \frac{x}{\varepsilon}\right)\right\|_{L^{2}(\partial \Omega)} \lesssim\left\|\nabla u_{0}\right\|_{L^{2}(\partial \Omega)} \lesssim\left\|u_{0}\right\|_{H^{2}(\Omega)} .
$$

We deduce from this that

$$
\left\|u_{\varepsilon}(x)-u_{0}(x)\right\|_{L^{2}} \lesssim \varepsilon\left\|u_{0}\right\|_{H^{2}}
$$

as required.

Proof of Theorem 6.3. We show that

$$
\varepsilon\left\|\nabla \theta_{\varepsilon}(x)\right\|_{L^{2}} \lesssim \sqrt{\varepsilon}
$$

from which (6.20) follows immediately. But

$$
\begin{aligned}
\varepsilon\left\|\nabla \theta_{\varepsilon}(x)\right\|_{L^{2}} & \lesssim \varepsilon\left\|u_{1}\left(x, \frac{x}{\varepsilon}\right)\right\|_{H^{1 / 2}(\partial \Omega)} \\
& \lesssim \varepsilon\left\|u_{1}\left(x, \frac{x}{\varepsilon}\right)\right\|_{L^{2}(\partial \Omega)}^{1 / 2}\left\|\nabla u_{1}\left(x, \frac{x}{\varepsilon}\right)\right\|_{L^{2}(\partial \Omega)}^{1 / 2} \\
& \lesssim \varepsilon \times\left(\frac{1}{\varepsilon}\right)^{1 / 2}
\end{aligned}
$$


where we have used interpolation for the second line and the fact that

$$
\left\|\nabla u_{1}\left(x, \frac{x}{\varepsilon}\right)\right\|_{L^{2}(\partial \Omega)} \lesssim \frac{1}{\varepsilon} .
$$

\subsubsection{Global error}

We combine here the results of both of the last sections. Namely, putting together the finite element approximation error and the homogenization error, one proves the following theorem.

Theorem 6.5. With the preceding notation, we have

$$
\left\|u_{\varepsilon}-u_{0, h}\right\|_{L^{2}} \lesssim(\varepsilon+h)\left\|u_{0}\right\|_{H^{2}} .
$$

Proof. The proof is quite obvious and simply relies on the fact that by the triangle inequality, one has

$$
\begin{aligned}
\left\|u_{\varepsilon}-u_{0, h}\right\|_{L^{2}} & \leq\left\|u_{\varepsilon}-u_{0}\right\|_{L^{2}}+\left\|u_{0}-u_{0, h}\right\|_{L^{2}} \\
& \leq\left(C_{1} \varepsilon+C_{2} h\right)\left\|u_{0}\right\|_{H^{2}} .
\end{aligned}
$$

The first estimation is a consequence of homogenization theory while the second one follows from Lemma 6.4.

It is important to notice that there is no better estimation of $\left\|u_{0}-u_{0, h}\right\|_{L^{2}}$ in contrast to the situation of AubinNitsche's lemma. This is due to the fact that $u_{0, h}$ solves a problem which is only a $h^{k}$ approximation of the problem solved by $u_{0}$.

Turning now to the $H^{1}$ norm, we have

Theorem 6.6. With the preceding notation, we have

$$
\left\|u_{\varepsilon}-u_{0, h}-\varepsilon u_{1, h}\left(x, \frac{x}{\varepsilon}\right)\right\|_{H^{1}} \lesssim\left(\sqrt{\varepsilon}+h^{k}\right)\left\|u_{0}\right\|_{H^{2}} .
$$

Proof. The proof is also a simple use of the preceding estimates. Indeed

$$
\begin{aligned}
\left\|u_{\varepsilon}-u_{0, h}-\varepsilon u_{1, h}\left(x, \frac{x}{\varepsilon}\right)\right\|_{H^{1}} \leq & \left\|u_{\varepsilon}-u_{0}-\varepsilon u_{1}\left(x, \frac{x}{\varepsilon}\right)\right\|_{H^{1}}+\left\|u_{0}-u_{0, h}\right\|_{H^{1}} \\
& +\varepsilon\left\|u_{1}\left(x, \frac{x}{\varepsilon}\right)-u_{1, h}\left(x, \frac{x}{\varepsilon}\right)\right\|_{H^{1}} .
\end{aligned}
$$

We already know that

$$
\left\|u_{\varepsilon}-u_{0}-\varepsilon u_{1}\left(x, \frac{x}{\varepsilon}\right)\right\|_{H^{1}} \lesssim \sqrt{\varepsilon}\left\|u_{0}\right\|_{H^{2}}
$$

and

$$
\left\|u_{0}-u_{0, h}\right\|_{H^{1}} \lesssim h^{k}\left\|u_{0}\right\|_{H^{2}} .
$$

Therefore it remains to estimate the last term. But since the correctors $\omega_{i}$ are uniformly bounded, it is easily seen that

$$
\varepsilon\left\|u_{1}\left(x, \frac{x}{\varepsilon}\right)-u_{1, h}\left(x, \frac{x}{\varepsilon}\right)\right\|_{L^{2}} \lesssim \varepsilon\left\|u_{0}-u_{0, h}\right\|_{H^{1}} \lesssim \varepsilon h^{k}\left\|u_{0}\right\|_{H^{2}} .
$$

Eventually,

$$
\varepsilon\left\|u_{1}\left(x, \frac{x}{\varepsilon}\right)-u_{1, h}\left(x, \frac{x}{\varepsilon}\right)\right\|_{H^{1}} \lesssim\left\|u_{0}-u_{0, h}\right\|_{H^{1}} \lesssim h^{k}\left\|u_{0}\right\|_{H^{2}},
$$

which leads to the result.

A better approximation is obtained with the use of the boundary layer $\theta_{\varepsilon}$. However, these correctors are not obvious to compute since they involve the operator with the oscillating coefficients which probably make them seldom used in practice. These details are outside the scope of the present notes and we refer the interested reader to [12] for more details on this subject.

\subsection{The multiscale finite element method (MFEM)}

The multiscale finite element method has a wider range of application than the preceding method. In particular, it easily applies to the case where the diffusion tensor $A$ not only depends on the fast variable $y=\frac{x}{\varepsilon}$ but also on the slow variable $x$. The goal is still to try to catch the highly oscillating solutions (at scale $\varepsilon$ ) with a mesh of size $h \gg \varepsilon$, but, as we shall see, there is no need to solve analytically the homogenized problem. 


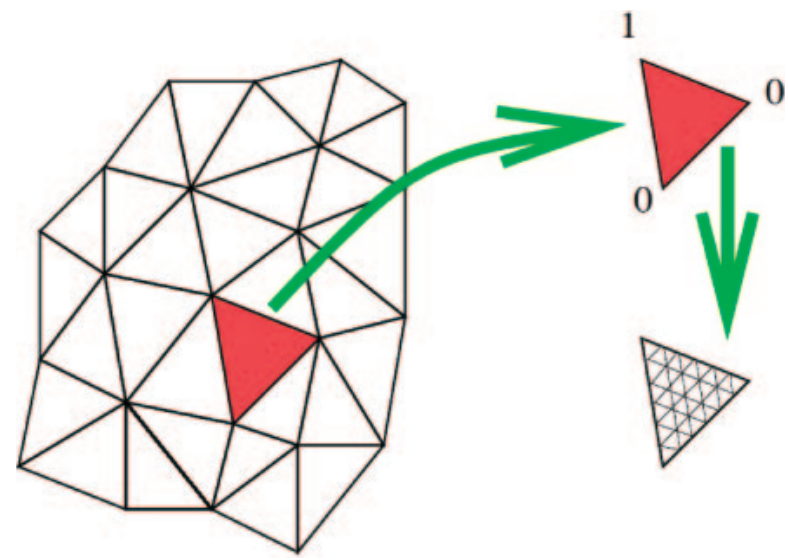

Fig. 6.1. The multiscale Finite Element Method. On the mesh, one computes the base functions as oscillating solutions on a thinner mesh.

\subsubsection{Description of the method}

The idea behind the multiscale finite element method is to precompute base functions that are oscillating. In the sequel we work for simplicity with finite elements of low order $k=1$ but there is no intrinsic difficulty to extend the method to higher degrees. We therefore consider a triangulation $\mathcal{T}_{h}$ and the $P^{1}$ base functions $\left(\phi_{i}\right)_{1 \leq i \leq N}$ which are globally continuous on $\mathcal{T}_{h}$, affine on each triangle $K \subset \mathcal{T}_{h}$ functions and satisfy

$$
\phi_{i}\left(x_{j}\right)=\delta_{i j} \text {, }
$$

where $\left(x_{j}\right)_{1 \leq j \leq N}$ are the vertices of the mesh and $\delta_{i j}$ is the Kronecker symbol. As we have already seen, solving the discrete variational formulation with functions in the discrete space $V_{h}=\operatorname{vect}\left\{\phi_{i}\right\}$ does not lead to a good error estimate because roughly speaking the base functions $\phi_{i}$ do not see the scale of the oscillations. The idea of the multiscale finite element method is therefore to solve the classical variational formulation but with a different set of base functions $\left(\psi_{i}\right)_{1 \leq i \leq N}$ which take into account the oscillations of the coefficients. More precisely we compute $\psi_{i}$ such that for any triangle $K \subset \mathcal{T}_{h}$,

$$
\left\{\begin{array}{l}
-\operatorname{div}\left(A\left(\frac{x}{\varepsilon}\right) \nabla \psi_{i}\right)=0 \text { in } K, \\
\psi_{i}=\phi_{i} \text { on } \partial K .
\end{array}\right.
$$

Of course it is easily seen that $\left.\psi_{i}\right|_{K}=0$ if $x_{i}$ is not a vertex of $K$. Using the base functions $\left(\psi_{i}\right)_{1 \leq i \leq N}$, we then solve the discrete variational formulation as usual, namely, we set $W_{h}=\operatorname{vect}\left\{\psi_{i}\right\}$ and solve

$$
\text { Find } u_{h} \in W_{h} \text { such that } \forall v_{h} \in W_{h}, \int_{\Omega} A\left(\begin{array}{l}
x \\
\varepsilon
\end{array}\right) \nabla u_{h} \cdot \nabla v_{h} d x=\int_{\Omega} f v_{h} d x \text {. }
$$

Before turning to the error analysis, let us emphasize the numerical difficulties posed by the method from a practical viewpoint. It should be remarked that the method is rather direct and straightforward beside two challenging tasks:

- The computation of the base functions $\psi_{i}$ on each triangle needs a finer mesh which is capable of catching the oscillations and, therefore, whose mesh size is of order $\varepsilon$. Although this seems difficult, these tasks are completely independent from one triangle to another and thus the problem could be solved in parallel.

- At the end, the problem that needs to be solved has a size which is equal to $N$ (the number of vertices in the mesh). It is therefore reasonable.

- We also notice that, although the multiscale finite element method does not need $A$ to be periodic in $Y$, the error analysis given in the following section assumes this for simplicity.

\subsubsection{Error analysis of the MFEM}

The main theorem that we want to show in this section is the following.

Theorem 6.7. Let $u_{\varepsilon}$ be the solution of the continuous problem (6.1), and $u_{h}$ the multiscale finite element solution (6.31). Then one has the estimation

$$
\left\|u_{\varepsilon}-u_{h}\right\|_{H^{1}} \lesssim h\|f\|_{L^{2}}+\left(\frac{\varepsilon}{h}\right)^{1 / 2}
$$


Before proceeding to the proof, let us remark that the first term is the classical error obtained for a finite element method applied to a regular problem and is independent of $\varepsilon$. However, the second term is not classical and clearly shows that $h$ must not be too small and in other words, non comparable to $\varepsilon$. This is the so-called resonance phenomenon (between $h$ and $\varepsilon$ ) in the literature.

Proof. The proof follows several steps. We first remark that it is a consequence of Céa's lemma and the interpolation error estimation given below.

Lemma 6.5 (interpolation error). Let $u_{\varepsilon}$ be the solution of the continuous problem (6.1), and $u_{I}$ the multiscale interpolant of the homogenized solution $u_{0}$ with the base functions $\psi_{i}$

$$
u_{I}(x)=\sum_{i=1}^{N} u_{0}\left(x_{i}\right) \psi_{i}(x) .
$$

Then one has the estimate

$$
\left\|u_{\varepsilon}-u_{I}\right\|_{H^{1}} \lesssim h\|f\|_{L^{2}}+\left(\frac{\varepsilon}{h}\right)^{1 / 2}
$$

Proof. Since $\psi_{i}$ solve (6.30), we can make the multiscale expansion of $\psi_{i}$ on any triangle $K$ of the triangulation. This gives

$$
\psi_{i} \sim \psi_{i}^{0}+\varepsilon \psi_{i}^{1}-\varepsilon \theta_{i}^{1}
$$

where $\psi_{i}^{0}, \psi_{i}^{1}$, and $\theta_{i}^{1}$ are solution to

$$
\begin{aligned}
-\operatorname{div}\left(\bar{A} \nabla \psi_{i}^{0}\right) & =0 \text { on } K \\
\psi_{i}^{0} & =\phi_{i} \text { on } \partial K \\
\psi_{i}^{1} & =-\sum_{j=1}^{2} \omega_{j} \frac{\partial \psi_{i}^{0}}{\partial x_{j}} \\
-\operatorname{div}\left(A\left(\frac{x}{\varepsilon}\right) \nabla \theta_{i}^{1}\right) & =0 \text { on } K \\
\theta_{i}^{1} & =\psi_{i}^{1} \text { on } \partial K
\end{aligned}
$$

$\left(\omega_{j}\right.$ are the corrector functions defined on the unit cell $Y$ ). Notice that (6.33) and (6.34) imply that actually $\psi_{i}^{0}=\phi_{i}$. By linearity of (6.32), we get a similar expression for $u_{I}$

$$
u_{I} \sim u_{I}^{0}+\varepsilon u_{I}^{1}-\varepsilon \theta_{I}^{1}
$$

with

$$
\begin{aligned}
-\operatorname{div}\left(\bar{A} \nabla u_{I}^{0}\right) & =0 \text { on } K \\
u_{I}^{1} & =-\sum_{j=1}^{2} \omega_{j} \frac{\partial u_{I}^{0}}{\partial x_{j}} \\
-\operatorname{div}\left(A\left(\frac{x}{\varepsilon}\right) \nabla \theta_{I}^{1}\right) & =0 \text { on } K \\
\theta_{I}^{1} & =u_{I}^{1} \text { on } \partial K .
\end{aligned}
$$

Now, we remark that $u_{I}^{0}$ is the classical affine interpolant of $u_{0}$ (indeed $\left.u_{I}^{0}(x)=\sum_{i=1}^{N} u_{0}\left(x_{i}\right) \psi_{i}^{0}(x)=\sum_{i=1}^{N} u_{0}\left(x_{i}\right) \phi_{i}(x)\right)$. We now make use of the following lemma.

Lemma 6.6. With the above notations, we have

$$
\left\|u_{I}-u_{I}^{0}-\varepsilon u_{I}^{1}+\varepsilon \theta_{I}^{1}\right\|_{H^{1}} \lesssim \varepsilon\|f\|_{L^{2}} .
$$

Proof of Lemma 6.6. We know from the previous theory, and more precisely Theorem 6.4 that on each triangle $K$ of the triangulation one has

$$
\left\|u_{I}-u_{I}^{0}-\varepsilon u_{I}^{1}+\varepsilon \theta_{I}^{1}\right\|_{H^{1}(K)} \lesssim \varepsilon\left\|u_{I}^{0}\right\|_{H^{2}(K)}=\varepsilon\left\|u_{I}^{0}\right\|_{H^{1}}
$$

since $u_{I}^{0}$ solves (6.35). Squaring this inequality and summing over all the triangles $K$ of the triangulation gives

$$
\left\|u_{I}-u_{I}^{0}-\varepsilon u_{I}^{1}+\varepsilon \theta_{I}^{1}\right\|_{H^{1}} \lesssim \varepsilon\left\|u_{I}^{0}\right\|_{H^{1}} \lesssim \varepsilon\|f\|_{L^{2}} .
$$


Now, we make the multiscale expansion of $u_{\varepsilon}$

$$
u_{\varepsilon} \sim u^{0}+\varepsilon u^{1}-\varepsilon \theta^{1}
$$

where we know (still from Theorem 6.4)

$$
\left\|u_{\varepsilon}-u^{0}-\varepsilon u^{1}+\varepsilon \theta^{1}\right\|_{H^{1}} \lesssim \varepsilon\left\|u^{0}\right\|_{H^{2}} \lesssim \varepsilon\|f\|_{L^{2}} .
$$

We therefore deduce that

$$
\left\|u_{\varepsilon}-u_{I}\right\|_{H^{1}} \lesssim\left\|u^{0}-u_{I}^{0}\right\|_{H^{1}}+\varepsilon\left\|u^{1}-u_{I}^{1}\right\|_{H^{1}}+\varepsilon\left\|\theta^{1}-\theta_{I}^{1}\right\|_{H^{1}}+\varepsilon\|f\|_{L^{2}} .
$$

It remains to estimate the three terms of the right hand side. But we already know that

$$
\left\|u^{0}-u_{I}^{0}\right\|_{H^{1}} \lesssim h\|f\|_{L^{2}}
$$

from the classical finite element theory. Next, notice that the correctors $\omega_{i}$ are regular functions that satisfy $\omega_{i} \in W^{1, \infty}(Y)$. We therefore have

$$
\varepsilon\left\|u^{1}-u_{I}^{1}\right\|_{L^{2}(K)} \lesssim \varepsilon\left\|\nabla\left(u^{0}-u_{I}^{0}\right)\right\|_{L^{2}(K)} \lesssim \varepsilon h\left\|u^{0}\right\|_{H^{2}(K)}
$$

which after summation over all the triangles leads to

$$
\varepsilon\left\|u^{1}-u_{I}^{1}\right\|_{L^{2}} \lesssim \varepsilon\left\|\nabla\left(u^{0}-u_{I}^{0}\right)\right\|_{L^{2}} \lesssim \varepsilon h\left\|u^{0}\right\|_{H^{2}} \lesssim \varepsilon h\|f\|_{L^{2}} .
$$

On the oher hand, one has

$$
\begin{aligned}
& \varepsilon\left\|\nabla\left(u^{1}-u_{I}^{1}\right)\right\|_{L^{2}(K)}= \varepsilon\left\|\nabla\left(\sum_{j=1}^{2} \omega_{j}\left(\frac{\partial u^{0}}{\partial x_{j}}-\frac{\partial u_{I}^{0}}{\partial x_{j}}\right)\right)\right\|_{L^{2}(K)} \\
& \lesssim \varepsilon \sup _{j}\left(\left\|\nabla \omega_{j}(x / \varepsilon)\right\|_{L^{\infty}(K)}\left\|\frac{\partial u^{0}}{\partial x_{j}}-\frac{\partial u_{I}^{0}}{\partial x_{j}}\right\|_{L^{2}(K)}\right) \\
&+\varepsilon \sup _{j}\left(\left\|\omega_{j}(x / \varepsilon)\right\|_{L^{\infty}(K)}\left\|\frac{\partial u^{0}}{\partial x_{j}}-\frac{\partial u_{I}^{0}}{\partial x_{j}}\right\|_{H^{1}(K)}\right) \\
& \lesssim\left\|\nabla\left(u^{0}-u_{I}^{0}\right)\right\|_{L^{2}(K)}+\varepsilon\left\|\nabla\left(u^{0}-u_{I}^{0}\right)\right\|_{H^{1}(K)} .
\end{aligned}
$$

Summing again over the triangulation gives

$$
\varepsilon\left\|\nabla\left(u^{1}-u_{I}^{1}\right)\right\|_{L^{2}} \lesssim(h+\varepsilon)\left\|u^{0}\right\|_{H^{2}} \lesssim(h+\varepsilon)\|f\|_{L^{2}} .
$$

For the last term, we use the previous estimation of the correctors $\theta$. Namely

$$
\varepsilon\left\|\theta^{1}-\theta_{I}^{1}\right\|_{H^{1}} \leq \varepsilon\left\|\theta^{1}\right\|_{H^{1}}+\varepsilon\left\|\theta_{I}^{1}\right\|_{H^{1}} .
$$

On the one hand, we know that $\left\|\theta^{1}\right\|_{H^{1}} \lesssim\left\|u^{1}\right\|_{H^{1 / 2}(\partial \Omega)} \lesssim \varepsilon^{-1 / 2}$, while on each triangle $K$, one has $\left\|\theta_{I}^{1}\right\|_{H^{1}(K)} \lesssim$ $\left\|u_{I}^{1}\right\|_{H^{1 / 2}(\partial K)}$ and from interpolation

$$
\left\|u_{I}^{1}\right\|_{H^{1 / 2}(\partial K)}^{2} \lesssim\left\|u_{I}^{1}\right\|_{L^{2}(\partial K)}\left\|\nabla u_{I}^{1}\right\|_{L^{2}(\partial K)} .
$$

But, $\left\|u_{I}^{1}\right\|_{L^{2}(\partial K)} \lesssim\left\|\nabla u_{I}^{0}\right\|_{L^{2}(\partial K)} \lesssim h^{1 / 2}$ and $\left\|\nabla u_{I}^{1}\right\|_{L^{2}(\partial K)} \lesssim \varepsilon^{-1}\left\|\nabla u_{I}^{0}\right\|_{L^{2}(\partial K)} \lesssim h^{1 / 2} \varepsilon^{-1}$. We therefore deduce that

$$
\begin{aligned}
\varepsilon\left\|\theta_{I}^{1}\right\|_{H^{1}} & \lesssim \varepsilon\left(\sum_{K \in \tau_{h}}\left(h^{1 / 2} h^{1 / 2} \varepsilon^{-1}\right)\right)^{\frac{1}{2}} \\
& \lesssim \sqrt{\frac{\varepsilon}{h}}
\end{aligned}
$$

since there are $O\left(1 / h^{2}\right)$ triangles in the mesh.

Putting all the pieces together leads to

$$
\left\|u_{\varepsilon}-u_{I}\right\|_{H^{1}} \lesssim h\|f\|_{L^{2}}+\varepsilon h\|f\|_{L^{2}}+(h+\varepsilon)\|f\|_{L^{2}}+\sqrt{\varepsilon}+\sqrt{\frac{\varepsilon}{h}}
$$

which is the desired result in view of $\varepsilon \ll h \ll 1$.

The estimate given above shows a new feature. Indeed, if $h$ is of the order of $\varepsilon$, then the estimate breaks down. This phenomenon, usually called resonance implies that $\varepsilon$ must be small compared to $h$. Although this is clearly the goal of the method (to catch oscillations at a much finer scale than the mesh-scale) one must pay attention to this in practice. Many more details can be found in [10]. 


\subsection{Conclusion}

We have presented a small tour on the estimation for finite element method for homogenization problems. Applying the classical finite element method to the original problem is likely to fail due to the fine scale of the oscillations. Instead one can either compute the 2-scale limit (at order 1 and $\varepsilon$ ), or use the so-called multiscale finite element method for which the base functions are recomputed on each triangle of the triangulation. The extra work leads to much better error estimates.

\section{Bibliography}

[1] Allaire, G., "Homogenization and two-scale convergence," SIAM J. Math. Anal., 23(6): 1482-1518 (1992).

[2] Allaire, G., "Homogenization of the Stokes flow in a connected porous medium," Asymptotic Analysis, 2: 203-222 (1989).

[3] Braess, D., Finite Elements: Theory, Fast Solvers, and Applications in Solid Mechanics, Cambridge University Press (2007).

[4] Braides, A. and Defranceschi, A., Homogenization of Multiple Integrals, Oxford University Press (1998).

[5] Braides, A., "Gamma-Convergence for Beginners," Oxford Lecture Series in Mathematics and Its Applications 22, Clarendon Press (2002).

[6] Brenner, S. C. and Scott, R., "The mathematical theory of finite element methods, 3rd edition," Texts in Applied Mathematics, Springer (2002).

[7] Brezis, H., "Analyse fonctionnelle: Théorie et Applications," Mathématiques Appliquées pour la Maîtrise, Masson.

[8] Dal Maso, G., "An introduction to $\Gamma$-convergence," Progress in Nonlinear Differential Equations and their Applications, Birkhauser Boston (1993).

[9] De Giorgi, E., and Franzoni, T., "Su un tipo di convergenza variazionale," Atti Accad. Naz. Lincei Rend. Cl. Sci. Fis. Mat. Natur., 58(8): 0 (1975).

[10] Hou, T., Wu, X.-H., and Cai, Z., "Convergence of a multiscale finite element method for elliptic problems with rapidly oscillating coefficients,” Math. Comp., 68(227): 913-943 (1999).

[11] Keller, J. R., "Darcy's law for flow in porous media and the two-space method," Lecture Notes in Pure and Appl. Math., 54, Dekker, New-York (1980).

[12] Lions, J.-L., "Some methods in the mathematical analysis of systems and their control," Science Press, Beijing, Gordon and Breach, New York (1981).

[13] Lipton, R., and Avellaneda, M., "A Darcy law for slow viscous flow past a stationary array of bubbles," Proc. Roy. Soc. Edinburgh, 114A: 71-79 (1990).

[14] Moskow, S., and Vogelius, M., "First order corrections to the homogenized eigenvalues of a periodic composite medium. A convergence proof," Proc. Roy. Soc. Edinburg, 127: 1263-1295 (1997).

[15] Muntean, A., and Chalupecky, V., Homogenization Method and Multiscale Modeling, lecture notes.

[16] Nguetseng, G., "A general convergence result for a functional related to the theory of homogenization," SIAM J. Math. Anal., 20(3): 608-623 (1989)

[17] Sanchez-Palencia, E., "Non homogeneous media and vibration theory," Lecture notes in physics 127, Springer Verlag (1980).

[18] Tartar, L., Convergence of the homogenization process. Appendix of [17]. 